\title{
COVID-19 changing the face of the world. Can sub-Sahara Africa cope?
}

\author{
Mandidayingeyi H Machingauta ${ }^{1,2}$, Bwalya Lungu ${ }^{3}$, Edward M Lungu ${ }^{1}$ \\ ${ }^{1}$ Department of Mathematics and Statistical Sciences \\ Botswana International University of Science and Technology \\ Palapye, Botswana \\ hellen.machingauta@studentmail.biust.ac.bw, lungue@biust.ac.bw \\ ${ }^{2}$ Department of Surveying and Geomatics \\ Midlands State University, Gweru, Zimbabwe \\ pfupajenamh@staff.msu.ac.zw \\ ${ }^{3}$ Department of Food Science \\ University of California,Davis, CA 95616, USA \\ blungu@ucdavis.edu
}

Received: 7 September 2020, accepted: 11 March 2021, published: 29 March 2021

\begin{abstract}
We formulate a mathematical model for the spread of the coronavirus which incorporates adherence to disease prevention. The major results of this study are: first, we determined optimal infection coefficients such that high levels of coronavirus transmission are prevented. Secondly, we have found that there exists several optimal pairs of removal rates, from the general population of asymptomatic and symptomatic infectives respectively that can protect hospital bed capacity and flatten the hospital admission curve. Of the many optimal strategies, this study recommends the pair that yields the least number of coronavirus related deaths. The results for South Africa, which is better placed than the other sub-Sahara African countries, show that failure to address hygiene and adherence issues
\end{abstract}

will preclude the existence of an optimal strategy and could result in a more severe epidemic than the Italian COVID-19 epidemic. Relaxing lockdown measures to allow individuals to attend to vital needs such as food replenishment increases household and community infection rates and the severity of the overall infection.

Keywords-COVID-19; Hospital bed capacity; Removal rates; Optimal strategies

\section{INTRODUCTION}

The coronavirus pandemic has disrupted global economies and health systems in unprecedented ways. As of 20 January 2021 there were 96715656 recorded cases with 2068062 deaths

Copyright: (C) 2021 Machingauta et al. This article is distributed under the terms of the Creative Commons Attribution License (CC BY 4.0), which permits unrestricted use, distribution, and reproduction in any medium, provided the original author and source are credited.

Citation: Mandidayingeyi H Machingauta, Bwalya Lungu, Edward M Lungu, COVID-19 changing the

face of the world. Can sub-Sahara Africa cope?, Biomath 10 (2021), 2103117, 
and 69410634 recoveries, [34]. The coronavirus currently infecting humans for the first time started in China in November 2019 in the city of Wuhan. The virus is being referred to as SARS-CoV-2 to differentiate it from other coronaviruses, [33]. According to the Centers for Disease Control (CDC) the virus displays symptoms within $2-14$ days of exposure that may include fever, body aches, dry cough, fatigue, chills, headache, sputum production, myalgias, anorexia, sore throat, shortness of breath, loss of appetite and loss of smell. Most of these symptoms are similar to the symptoms of diseases caused by other pathogens such as bacteria (e.g tuberculosis, cholera etc), viruses (e.g influenza, HIV, SARS, MERS) and parasites (e.g malaria). In its severe form COVID-19 induces severe pneumonia and has led to high rates of death in about $1-5 \%$ of those infected [31]. However, some people infected with the virus may show little to no symptoms and are classified as asymptomatic carriers that are still able to transmit the virus to other individuals. These individuals play a major role in transmission as they are silent spreaders and it is challenging to determine the rate at which they spread the disease.

There are currently no vaccines or treatments available for COVID-19, but patients suffering from severe symptoms are usually hospitalized with median hospital stays of 10 to 13 days [9], [31], [37]. Most of the countries experiencing high numbers of COVID-19 cases are developed countries including the United States of America (USA), Italy, Spain, United Kingdom (UK), France, Germany, Russia, Brazil and China. Most of these developed countries have excellent health facilities. However, the COVID-19 epidemic in Italy, Spain, France and the USA has demonstrated that the current medical facilities were not designed to serve the populations during a pandemic. Moreover, the democratic system of governance which we have all cherished has been seriously challenged during these times when some form of authoritarian governance was required in order to enforce prevention measures such as social distancing, masks, lockdown etc [6], [21], [27], to control virus transmission.

The current Western health systems performed well during the initial stages of the coronavirus disease progression but have since been challenged due to the acute rise in infection rates. Various countries have had to make decisions over who is offered or not offered a bed in an Intensive Care Unit (ICU). The decisions were based on hospital bed capacity to avoid hospital overload and were dependent on early testing and isolating those who test positive as was the case in South Korea, Germany and China [16].

The epidemic in these developed countries has had very serious effects on the overall infrastructure and livelihoods of the people living there, and we believe that the effects on the sub-Sahara Africa region could very likely overwhelm this region where the economies are very weak and their ICU capacity compared to population sizes averages only 9 beds per 10000 inhabitants [32]. The current economic landscape will likely impact the ability of the various health systems in the long term to supply personal protective equipment (PPE) required by the frontline workers as they take care of infected patients.

The main mode of transmission of the virus is through person to person transmission and this can happen through droplets, airborne transmission, surface transmission and the fecal oral route. The required measures to stop the spread of COVID-19 include social distancing, face mask requirements for out of home activities, staying home if one feels sick or is nursing a cold. Good hygiene practices such as frequent washing of hands or not touching the face area especially the mouth, nose and eyes, good sanitary practices that include frequent sanitation of surfaces. Drastic measures have included mandatory shelter-ins or lockdowns. While these types of measures are not new and have been shown to work, they will likely present a different set of problems for sub-Sahara Africa. The problems sub-Sahara Africa will face will be compounded but not limited to the following:

The low standards of good sanitary and hygiene measures may present an environment that 
supports virus spread. Good sanitary and hygiene practices have been encouraged and used in previous epidemics for thousands of years. The availability of clean water and poor hygiene (sanitary) will likely fuel the sub-Sahara coronavirus epidemic as has been the case for other ongoing epidemics such as the Ebola and cholera. The Ebola [17] and cholera epidemics which remain unresolved will compete with the coronavirus for health facilities and workers. The current health facilities are already inadequate, for example the number of hospital beds 9 per 10000 people [32], will prove inadequate based on the higher hospitalization rates reported in Italy, France and Spain for individuals infected with the coronavirus [24]. The average family in the poorest country in sub-Sahara Africa lives on less than $\$ 1$ per day and most of what they earn goes towards food and other necessities. Most families will not be able to afford the cost of soap and sanitizers to maintain the high levels of hygiene required to control or stop the spread of COVID-19 [26]. As a result of inadequate water supplies and lack of sanitizers/soaps a large number of the population may not practice good sanitation and hygiene practices. In addition, public bathrooms if available, do not provide soaps and sanitizers as they are not considered an essential commodity. Therefore, it will be challenging to adequately implement the frequent hygienic hand washing and sanitation of environmental surfaces.

Social distancing and isolation of suspected/confirmed COVID-19 cases is essential to stop virus transmission and spread. However, the facilities required to effectively isolate infected individuals or individuals suspected to have been exposed to the virus are non-existent in sub-Sahara Africa. For example, most infected individuals or those exposed to the virus will not have the luxury of a separate bedroom with separate sanitary facilities in their homes. It is common to find 8 people living in a small two bed-roomed house, therefore it will be impossible to institute social distancing and isolation practices in the event of suspected exposure or infection.
As part of social distancing, shelter-in or lockdown procedures, limitations have been placed on our movements. We can go to the grocery store to purchase food and other essential products. However, there are limits to how many grocery trips we can make and this means that families will have to buy food in bulk and store it at home. Most of the food we eat such as fresh vegetables, fruits and meats are perishable and thus require refrigeration in order to extend their shelf life. Most people in sub-Sahara Africa do not have refrigerators and even those who have refrigerators are subjected to frequent power interruption and can only buy enough supplies for a few days. This means that frequent visits to the shops and open markets is unavoidable. Food supply, harvesting etc in most countries will be challenged as lockdowns are implemented to slowdown social interaction and consequently disease spread. We may start to see disruptions in the supply chain, a dip in demand for commodities, loss of income as a result of layoffs and cash flow issues. We have already observed the impact to the food and commodity distribution system. In the most impacted developed countries such as the USA, it has not been uncommon since early March to find empty shelves where commodities such as sanitizers, disinfectants, soaps, toilet paper, paper towels, rice and canned goods used to be found. As a result of the early panic, the demand of these goods exceeded the supply and shortages resulted. Such scenarios would likely become the norm in sub-Sahara Africa. Developed countries such as Canada, Australia, Germany and the UK are paying their citizens to stay at home. The state of our economies in sub-Sahara Africa will not allow us to pay salaries and ensure every member of society has adequate supplies. People will have to calculate the risk of sure starvation if they obey the lockdown measures or risk going out and contracting COVID-19 because they have to make a living.

The World Health Organization (WHO) recently released guidance on the use of face masks, as a result countries are instituting rules requiring 
citizens to wear face masks from cloth material. In sub-Saharan countries, this has brought up questions of affordability of face masks and most of the population is waiting for government handouts of face masks. While it will be challenging to implement face mask wearing overall, it will be even more challenging to ensure face masks are washed prior to reuse and that people understand that face masks are not a $100 \%$ effective barrier. Therefore, people need to be educated on how the face masks should be worn and removed to avoid virus transmission and/or cross contamination as cloth masks are being removed. In addition face masks need to be used with all the hygiene and sanitary practices to control this virus. In light of all this, education of the masses is critical in the control of COVID-19 and needs to be a part of the complete COVID-19 mitigation framework.

African culture will play a significant role during this pandemic as is the case for Ebola and cholera epidemics. For example, most relatives of infected individuals will be unlikely to comply fully with the directives of social distancing and isolation. Adherence to precautionary measures in public places such as shops and open markets that are visited by people from different economic, educational and healthy backgrounds will be challenged (markets in Nigeria, Kenya, Tanzania, Burundi etc).

Behavioral norms are the most difficult to break. For example, young people are taught to cough into their fisted hands but now it is recommended to use disposable tissues that are a luxury to many or to cough into the elbow rather than hands [33]. The African culture is based on the use of hands, for example to greet, which makes it difficult to adhere to the new recommendation. Though the washing of hands during Ebola and cholera epidemics has been recommended, many people still walk out of the toilet without washing hands. It will be difficult for people during this pandemic to remember to wash their hands and wash them in the proper manner too [33].

Ubuntu (you are, because I am) is one of the cornerstones of African culture where a sense of community is still being practiced to a large extent. Social distancing becomes a challenge as Africans are very social people. For example, if one is unable to till land on their own, one can invite people in the village to help with the tilling whilst they provide beer and food for the helpers. Social gatherings such as funerals and weddings are held in high esteem. Limiting the number of people who can attend to limit social interactions is considered a taboo. In a typical household people go to different jobs and engage in different activities throughout the day and the question that comes uppermost is can social distancing be implemented in such situations?

Sub-Sahara African governments do not have the capacity to construct overflow hospital bed capacity.

This study will address the following objectives: First, to determine the total number of individuals likely to be infected with COVID-19. Secondly, to find possible hospitalization strategies that would not overload hospital bed capacity and the number of infected individuals who would need to be safely isolated. Finally, to find a strategy which yields the lowest number of deaths.

\section{DO PEOPLE LEARN FROM PREVIOUS EPIDEMICS: EBOLA}

To date Africa has registered very few cases of Covid-19 infections and deaths, even though the cases are rising. One wonders whether frequent exposure of the African population to epidemics has prepared it to prevention protocols. We give examples from Ebola in the Democratic Republic of Congo (DRC) and the Sudan, where prior exposure to the epidemic reduced the disease caseload.

The data for various epidemics in the DRC and the Sudan presented in Figures 1(a) and 1(b) shows a very high peak of total infections for the first epidemic and declining number of total infected during subsequent epidemics. The data suggests that the populations in the two countries have learnt how to manage an epidemic (Ebola) which requires high standards of hygiene and social distancing. Levy et al, [15] have shown that the severity of an outbreak is linked to the level 
(a)

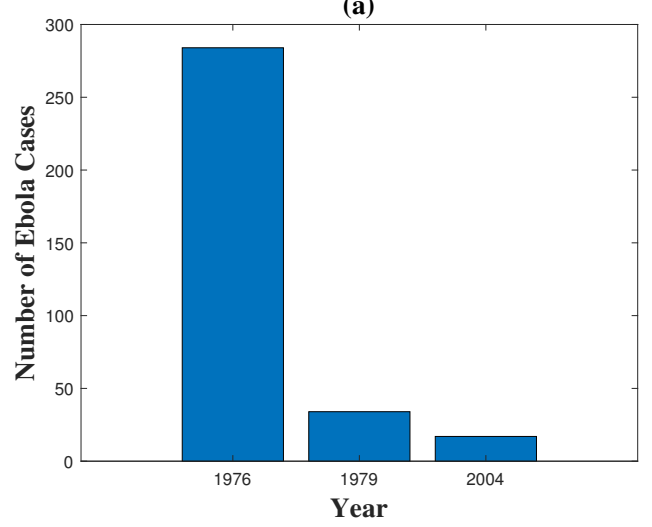

(b)

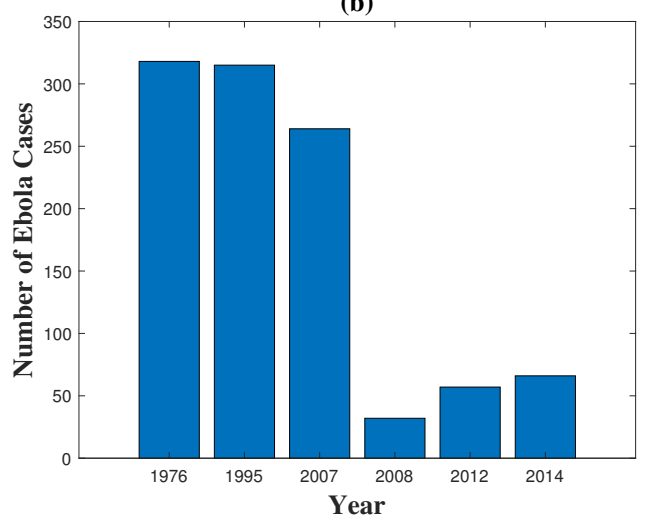

Fig. 1: Ebola outbreaks plots for (a) Sudan and (b) the Democratic Republic of Congo.

(a)

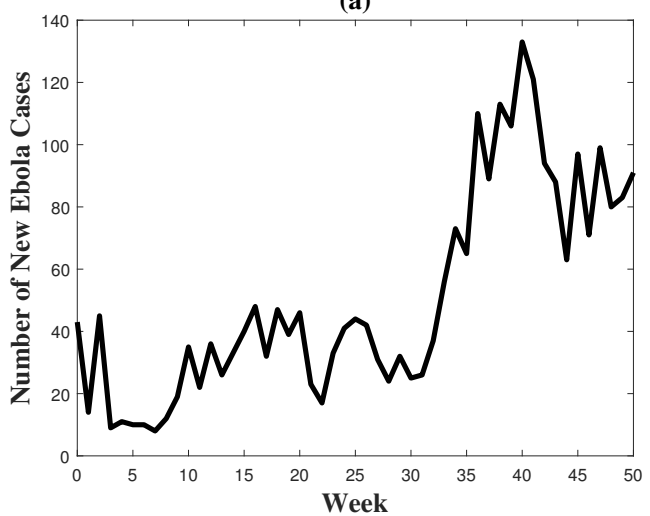

(b)

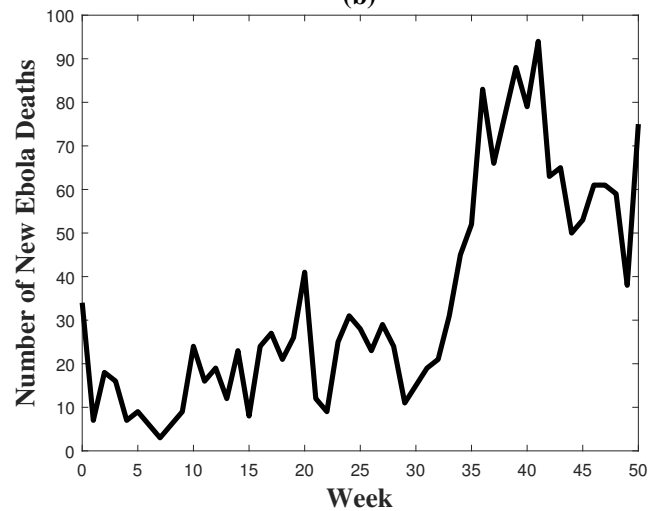

Fig. 2: Democratic Republic of Congo plots for (a) new Ebola cases (b) new Ebola deaths.

of prior knowledge and education of the general population as well as preparedness of health care facilities.

Using data from the Democratic Republic of Congo (DRC) for new Ebola cases and new Ebola deaths, we notice a trend of rising to a peak and then declining, indicating two phases

(i) naivety to the virus early in the infection and

(ii) experience towards adherence to prevention protocols (Figures 2(a) and 2(b)).

Ebola and COVID-19 management are similar in many ways. They both rely on very high standards of hygiene, avoiding hand shaking and low density occupancy in residential homes. The difficulty one encounters when modeling Ebola or COVID-19 is modeling the infections arising from poor hygiene, social distancing etc. From Figures 1 and 2, we suggest modeling infections from poor hygiene and lack of social distancing by a function fitted to the data and depicted in Figure 3. This function represents high infections due to poor understanding of the prevention measures early into the epidemic, but as the infection progresses individuals who apply the knowledge from previous epidemics adapt to avoid infections as was the case for Ebola. The function in Figure 3 mimics the process observed for Ebola and is adopted in this study.

\section{QUALITY OF THE LOCKDOWN}

Since SARS-2 is a respiratory disease, we want to incorporate the effects of household transmission due to an infected individual $i$, in household 


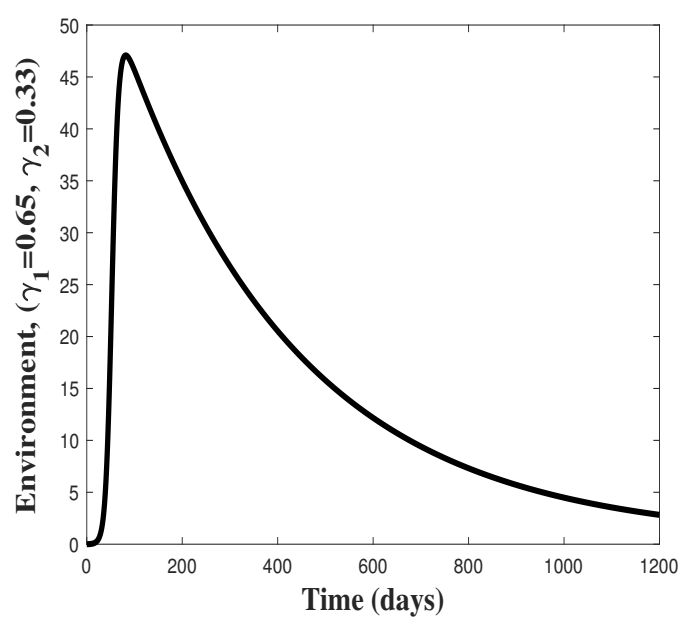

Fig. 3: Time course for the environment

$h$ at time $t$ during the lockdown. The rate of exposure from a member of the family $j$ to $i$ is given by:

$$
\nu_{h}=\eta_{g} \times \psi_{H} \times \psi_{i, \text { inf }}
$$

where $\psi_{H}$ depends on household size and $\psi_{i \text {,inf }}$ represents asymptomatic or symptomatic status of the infection. An individual in the household $h$ is also exposed to community transmission because a member of the household has to leave the house to transact with the community, for example to buy food or to go to work (if they are front-line workers) during the lockdown. This movement is inevitable in poverty stricken Africa where food insecurity is common and food must be sourced daily. The rate of community exposure is given by:

$$
C_{h}=\varepsilon_{g} \times f_{g}(t) \times \psi_{i, \text { age age group }}
$$

where $\varepsilon_{g}$ is the baseline exposure from the community, $f_{g}$ is a time dependent curve that modifies the community rate of exposure over time and

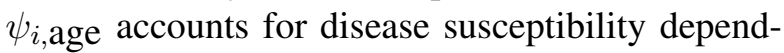
ing on age.

The rate of exposure of individual $i$ in household $h$ in which a member goes out to transact with the community is given by:

$$
\lambda=S_{i, g(t)}\left[M_{i, h(t)} \sum_{j \neq i} \nu_{h}+C_{h}\right]
$$

(3) has been used to moderate transmission of respiratory synctial virus which is similar to SARS2, [13]. The approach described in (1), (2) and (3) is described in detail in [13]. We have used the data from [13] to incorporate the quality of the lockdown and to explain why the number of COVID-19 cases exploded after the lockdown.

\section{MODEL DESCRIPTION}

We develop a simple model in the context of the sub-Sahara Africa environment which consists of a class of individuals, $S$, who are susceptible to the disease, a class of individuals, $E$, who have been exposed to the disease, a class of asymptomatic individuals, $I_{a s}$. These are individuals who are not showing symptoms but are transmitting the virus. A class of symptomatic infectives, $I_{s}$, a class of individuals who require hospitalization or to be isolated, $H$, and a class of recovered individuals, $R$. We note that $I_{a s}$ and $I_{s}$ can infect $S$ directly and indirectly by contaminating the environment $\phi$. For simplicity of notation, let

$$
\begin{aligned}
x(t) & =\left(x_{1}(t), x_{2}(t), x_{3}(t), x_{4}(t), x_{5}(t), x_{6}(t)\right), \\
& =\left(S(t), E(t), I_{a s}(t), I_{s}(t), H(t), R(t)\right), \\
x_{7}(t) & =\phi(t), \\
N(t) & =x_{1}(t)+x_{2}(t)+x_{3}(t)+x_{4}(t)+x_{5}(t)+x_{6}(t) .
\end{aligned}
$$

We consider the following model:

$$
\begin{aligned}
\frac{d x_{1}}{d t}= & \pi-\frac{\left(\beta_{1} x_{3}+\beta_{2} x_{4}\right) x_{1}}{N}-\beta_{3} x_{7} x_{1} \\
& -\mu x_{1}, \\
\frac{d x_{2}}{d t}= & \frac{\left(\beta_{1} x_{3}+\beta_{2} x_{4}\right) x_{1}}{N}+\beta_{3} x_{7} x_{1} \\
& -\left(\alpha_{1}+\mu\right) x_{2}, \\
\frac{d x_{3}}{d t}= & \alpha_{1} x_{2}-\left(\gamma_{1}+\alpha_{2}+\mu+\kappa_{1}\right) x_{3},
\end{aligned}
$$


M H Machingauta, B Lungu, E M Lungu, COVID-19 changing the face of the world. Can sub-Sahara ...

$$
\begin{aligned}
\frac{d x_{4}}{d t} & =\alpha_{2} x_{3}-\left(\gamma_{2}+\delta_{1}+\kappa_{2}\right) x_{4}, \\
\frac{d x_{5}}{d t} & =\gamma_{1} x_{3}+\gamma_{2} x_{4}-\left(\delta_{2}+\kappa_{3}\right) x_{5}, \\
\frac{d x_{6}}{d t} & =\kappa_{1} x_{3}+\kappa_{2} x_{4}+\kappa_{3} x_{5}-\mu x_{6},
\end{aligned}
$$

where

$$
(\pi, \mu)= \begin{cases}(0,0) & \text { during lockdown } \\ \neq(0,0) & \text { no lockdown, inter-zonal } \\ & \text { movement allowed }\end{cases}
$$

For short lockdown periods one can assume that $\mu=0$. This assumption is not valid for long lockdowns. The quality of the environment is described by the equation:

$$
\frac{d x_{7}}{d t}=\frac{\alpha_{3}\left(x_{3}+x_{4}\right)}{N}-\mu_{1} x_{7}
$$

The parameter $\alpha_{3}$ measures the rate of contaminating the environment, that is, the rate at which the amount of pathogens are released into the environment by both asymptomatic and symptomatic infectives. Equation (11) is also supported by Berge et al. [2] for their model on Ebola. The model flow diagram is given below:

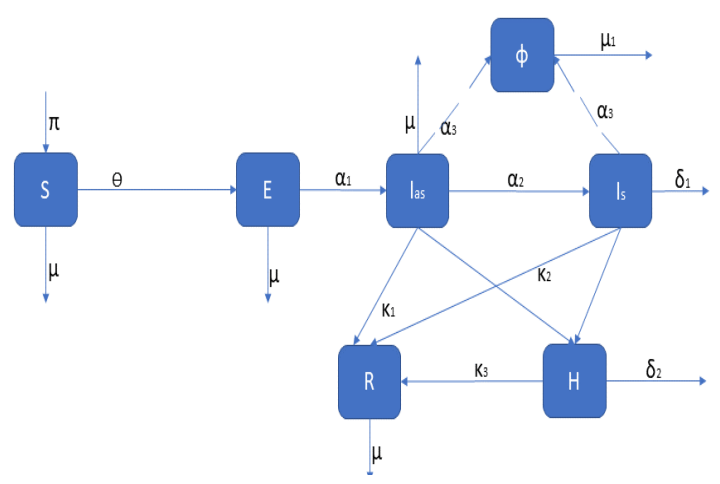

Fig. 4: Model flow diagram

where $\theta=\frac{\left(\beta_{1} x_{3}+\beta_{2} x_{4}\right) x_{1}}{N}+\beta_{3} x_{7} x_{1}$.

Equation (4) describes the rate of change in the susceptible population, $x_{1}$. The first term in (4) represents recruitment of people into the susceptible class through movement of people from different zones when this is allowed. This term is zero during a lockdown but may be nonzero if inter-zone movements are allowed. The second term represents loss due to infection of susceptible individuals by asymptomatic infectives, $x_{3}$, at the rate $\beta_{1}$ and symptomatic infectives, $x_{4}$, at the rate $\beta_{2}$. The third term represents indirect infections due to an unclean environment at the rate $\beta_{3}$. As time has progressed, it has become necessary to account for loss due to other causes such as natural death at the rate $\mu$. There has been confusion over the number of covid deaths and it has become pathologically necessary to ascertain that a covid infected individual actually died of covid complications, [22], [30]. However, $\mu$ was ignored during early stages of the pandemic and every covid infected individual was assumed to have died of covid.

Equation (5) describes the rate of change of the exposed class, $x_{2}$. The first two terms represent gain from infection of susceptible individuals and the third term represents loss due to sero-conversion to the asymptomatic state at the rate $\alpha_{1}$ and natural death at a constant rate $\mu$, respectively. The same comment regarding $\mu$ in equation (4) applies in this case.

Equation (6) describes the rate of change of the asymptomatic infected class, $x_{3}$. The first term represents gain from exposed individuals who are converting to sero-positive status without exhibiting symptoms. The second term represents loss due to conversion to symptomatic state and hospitalization at the rates $\alpha_{2}$ and $\gamma_{1}$, respectively. $\kappa_{1}$ represents losses from this class due to recovery and $\mu$ represents natural death as explained in (10). The first term on the right hand side of equation (7) represents gain from the asymptomatic state. The second term represents loss due to hospitalization or isolation and a blanket term representing loss due to both natural death and disease induced death at the rates $\gamma_{2}$ and $\delta_{1}=\left(\mu+\delta_{x_{4}}\right)$, respectively. Loss from this class due to recovery is assumed to occur at a constant rate $\kappa_{2}$.

Equation (8) describes the rate of change of the hospitalized and isolated class. The first two terms represent gain from testing and contact tracing of 
both asymptomatic and symptomatic individuals. The third term represents loss due to blanket death at the rate $\delta_{2}=\left(\mu+\delta_{x_{5}}\right)$ and recovery at the rate $\kappa_{3}$.

Equation (9) describes the rate of change of the recovered individuals. The first three terms represent gain from recovery of asymptomatic, symptomatic and hospitalized cases. The recovered class loses people through natural death at a rate $\mu$ for a long pandemic.

Equation (11) describes the rate of contaminating the environment due to the release of pathogens into the environment by symptomatic and asymptomatic infectives at the rate $\alpha_{3}$. The second term represents cleaning of the environment (naturally or due to interventions) at the rate $\mu_{1}$.

\section{Model Analysis}

\section{A. Positivity of solutions}

Denote by $\Re_{+}^{6}$ the points $x(t)=$ $\left(x_{1}(t), x_{2}(t), x_{3}(t), x_{4}(t), x_{5}(t), x_{6}(t)\right) \quad$ in $\Re^{6}$ with positive coordinates and consider the system (4) - (9) with initial values

$$
x^{0}=\left(x_{1}^{0}, x_{2}^{0}, x_{3}^{0}, x_{4}^{0}, x_{5}^{0}, x_{6}^{0}\right) \in \Re_{+}^{6} .
$$

We can state the following Lemma:

Lemma 1. If $x_{i}^{0} \geq 0, i=1, \ldots, 6$ then $x_{i}(t) \geq 0$ for $t>0, i=1, \ldots, 6$.

\section{Proof:}

First, we want to show that $0 \leq x_{7}(t) \leq \frac{\alpha_{3}}{\mu_{1}}$ where the lower bound signifies a clean environment and the upper bound signifies a Covid-19 contaminated environment. From (11), we have

$$
\begin{aligned}
\frac{d x_{7}}{d t} & =\frac{\alpha_{3}\left(x_{3}+x_{4}\right)}{N}-\mu_{1} x_{7} \\
& \leq \alpha_{3}-\mu_{1} x_{7} .
\end{aligned}
$$

The solution for a totally contaminated COVID-19 environment is given by

$$
x_{7}(t) \leq x_{7}(0) e^{-\mu_{1} t}+\frac{\alpha_{3}}{\mu_{1}}\left(1-e^{-\mu_{1} t}\right) .
$$

As

$$
t \rightarrow \infty, x_{7}(t) \leq \frac{\alpha_{3}}{\mu_{1}} .
$$

For a clean environment, (11) becomes

$$
\begin{aligned}
\frac{d x_{7}}{d t} & =\frac{\alpha_{3}\left(x_{3}+x_{4}\right)}{N}-\mu_{1} x_{7} \\
& \geq-\mu_{1} x_{7} \\
x_{7}(t) & =x_{0} e^{-\mu_{1} t} \geq 0 .
\end{aligned}
$$

Hence, we have

$$
0 \leq x_{7}(t) \leq \frac{\alpha_{3}}{\mu_{1}} .
$$

From equation (4), we have

$$
\begin{aligned}
\frac{d x_{1}}{d t}= & \pi-\frac{\left(\beta_{1} x_{3}+\beta_{2} x_{4}\right) x_{1}}{N}-\beta_{3} x_{7} x_{1} \\
& -\mu x_{1} \\
\geq & \pi-\left(\beta_{1}+\beta_{2}+\beta_{3} \frac{\alpha_{3}}{\mu_{1}}\right) x_{1} \\
\geq & -w x_{1}, \quad w=\left(\beta_{1}+\beta_{2}+\beta_{3} \frac{\alpha_{3}}{\mu_{1}}\right) .
\end{aligned}
$$

The solution is

$$
x_{1}(t) \geq x_{1}^{0} e^{-w t} \geq 0, \forall t \geq 0 .
$$

Similarly, we can show that for $i=2, \ldots, 6$

$$
x_{i}(t) \geq 0, i=2, \ldots, 6 .
$$

This completes the proof.

\section{B. Invariance}

The total population, $N(t)$, at time $t$ is given by

$$
\begin{aligned}
\frac{d N}{d t} & =\pi-\mu N-\delta_{x_{4}} x_{4}-\delta_{x_{5}} x_{5} \\
& \leq \pi-\mu N .
\end{aligned}
$$

By Gronwall inequality, it is easy to show that

$$
0 \leq N(t) \leq \frac{\pi}{\mu}
$$

For the existence of a unique bounded solution, we infer that any solution of the system (4) - (11) is non negative and bounded in $\Omega=$ $\left\{\left(x_{1}, x_{2}, x_{3}, x_{4}, x_{5}, x_{6}\right) \in \Re_{+}^{6}: N \leq \frac{\pi}{\mu}, x_{7} \leq \frac{\alpha_{3}}{\mu_{1}}\right\}$. 


\section{Disease Free Equilibrium (DFE)}

1) Disease Free Equilibrium for $\pi=0$ : During a strict lockdown, we can take $\pi=$ 0 . The system (4) - (11) has two equilibrium points, the disease eradication point $\zeta_{0}=$ $(0,0,0,0,0,0,0)$ and the disease endemic point $\zeta_{1}=\left(x_{1}^{*}, x_{2}^{*}, x_{3}^{*}, x_{4}^{*}, x_{5}^{*}, x_{6}^{*}, x_{7}^{*}\right)$. To establish the stability of $\zeta_{0}$, we use the Hartmann-Grobmann theorem which roughly states that near an equilibrium point, the dynamics of the original (nonlinear) system are the same as those for the linearized system. The linearized system for $(4)-(11)$ is given by:

$$
\frac{d y(t)}{d t}=A y(t)
$$

where

$$
y(t)=\left(x_{1}(t), x_{2}(t), x_{3}(t), x_{4}(t), x_{5}(t), x_{6}(t), x_{7}(t)\right)^{T}
$$$$
A=\left[\begin{array}{ccccccc}
0 & 0 & 0 & 0 & 0 & 0 & 0 \\
0 & -\alpha_{1} & 0 & 0 & 0 & 0 & 0 \\
0 & \alpha_{1} & -b_{11} & 0 & 0 & 0 & \\
0 & 0 & \alpha_{2} & -c_{11} & 0 & 0 & 0 \\
0 & 0 & \gamma_{1} & \gamma_{2} & -d_{11} & 0 & 0 \\
0 & 0 & \kappa_{1} & \kappa_{2} & \kappa_{3} & 0 & 0 \\
0 & 0 & \alpha_{3} & \alpha_{3} & 0 & 0 & -\mu_{1}
\end{array}\right]
$$

and

$$
\begin{aligned}
b_{11} & =\left(\gamma_{1}+\alpha_{2}+\kappa_{1}\right) \\
c_{11} & =\left(\gamma_{2}+\kappa_{2}+\delta_{1}\right) \\
d_{11} & =\left(\delta_{2}+\kappa_{3}\right)
\end{aligned}
$$

The eigenvalues of $|A-\lambda I|$ are given by $\left(0,0,-\alpha_{1},-b_{11},-c_{11},-d_{11},-\mu_{1}\right)$. The reproduction number $R_{0}$, is given by the largest spectral radius of the matrix $|A-\lambda I|$. In this instance the possible values of $R_{0}$ are $\max \left\{\alpha_{1}, b_{11}, c_{11}, d_{11}, \mu_{1}\right\}$. Since $\lambda_{i}<0, i=$ $1,2, \ldots, 7$, the system $(4)-(11)$ is stable and tends to $\zeta_{0}$ as $t \rightarrow \infty$. Provided the population maintains the covid control protocols and keeps $R_{0}$ below 1 , the disease will fail to establish in the population.
2) Disease Free Equilibrium for $\pi \neq 0$ : When inter-zone movements are allowed, that is, $\pi \neq 0$, the system $(4)-(11)$ has a disease free equilibrium point $\zeta_{0}=\left(\frac{\pi}{\mu}, 0,0,0,0,0,0\right)$.

We use the technique by Van den Driessche and Watmough [29] to find the model reproduction number. The matrix for new infections is given by $F$ whilst the matrix for other transitions is given by $V$, where

$$
\begin{gathered}
F=\left[\begin{array}{c}
\frac{\left(\beta_{1} x_{3}+\beta_{2} x_{4}\right) x_{1}}{N}+\beta_{3} x_{7} x_{1} \\
0 \\
0 \\
0
\end{array}\right], \\
V=\left[\begin{array}{c}
a x_{2} \\
b x_{3}-\alpha_{1} x_{2} \\
c x_{4}-\alpha_{2} x_{3} \\
\mu_{1} x_{7}-\frac{\alpha_{3}\left(x_{3}+x_{4}\right)}{N}
\end{array}\right]
\end{gathered}
$$

and

$$
\begin{aligned}
a & =(\alpha+\mu) \\
b & =\left(\gamma_{1}+\alpha_{2}+\kappa_{1}+\mu\right) \\
c & =\left(\gamma_{2}+\kappa_{2}+\delta_{1}\right) .
\end{aligned}
$$

The Jacobean matrices $\mathbf{F}$ of $F$ and $\mathbf{V}$ of $V$ are computed at the point $\zeta_{0}$ with respect to the infected classes $\left(x_{2}, x_{3}, x_{4}, x_{7}\right)$. Thus, the basic reproduction number $R_{0}$, given by the spectral radius of the matrix $\mathbf{F V}^{-1}$ is the maximum of the moduli of the eigenvalues of that matrix $\mathbf{F} \mathbf{V}^{-1}$ given by,

$$
R_{0}=R_{x_{3}}+R_{x_{4}}+R_{x_{7}}
$$

where,

$$
\begin{aligned}
R_{x_{3}} & =\frac{\alpha_{1} \beta_{1}}{a b} \\
R_{x_{4}} & =\frac{\alpha_{1} \alpha_{2} \beta_{2}}{a b c} \\
R_{x_{7}} & =\frac{\alpha_{1} \alpha_{3} \beta_{3}\left(c+\alpha_{2}\right)}{a b c \mu_{1}} .
\end{aligned}
$$

Based on $R_{0}$ we can state the following theorem:

Theorem 2. The DFE point, $\zeta_{0}$, is locally asymptotically stable if $R_{0}<1$ and unstable if $R_{0}>1$. 
Remark 1: $R_{0}$ can be greater than 1 even if $R_{x_{3}}<1, R_{x_{4}}<1, R_{x_{7}}<1$. It is necessary for each sub-reproduction number $R_{x_{3}}, R_{x_{4}}$ and $R_{x_{7}}$ to be less than 1 and their sum to be less than 1 for the disease to fail to establish in the population.

Remark 2: Unlike the case $\pi=0$, now the adherence to the covid protocols by each infected subpopulation, $x_{i}, i=3,4$, is more strict (adherence to social distancing, mask wearing etc) and the hygiene measures must be more strictly observed for the stability of $\zeta_{0}$ to be achieved.

3) Global stability of the Disease Free Equilibrium:

Theorem 3. The DFE point, $\zeta_{0}$, of system (4) (11) is globally asymptotically stable for $R_{0}<1$.

Proof. To prove theorem 3, we use KamgangSallet stability theorem, [10]. Let $Z=\left(Z_{1}, X_{2}\right)$ with $Z_{1}=\left(x_{1}, x_{6}\right) \in \Re^{2}$ and $Z_{2}=$ $\left(x_{2}, x_{3}, x_{4}, x_{5}, x_{7}\right) \in \Re^{5}$. In terms of $Z_{1}$ and $Z_{2}$ system (4) - (11) can be written as:

$$
\begin{aligned}
& \dot{Z}_{1}=A_{1}(Z)\left(Z_{1}-Z_{1}^{*}\right)+A_{12}(Z) Z_{2} \\
& \dot{Z}_{2}=A_{2}(Z)\left(Z_{2}\right)
\end{aligned}
$$

where $Z_{1}^{*}=\left(\frac{\pi}{\mu}, 0\right)$, with

$$
\begin{gathered}
A_{1}(Z)=\left[\begin{array}{cc}
-\mu & 0 \\
0 & -\mu
\end{array}\right] \\
A_{12}(Z)=\left[\begin{array}{ccccc}
0 & -\frac{\beta_{1} x_{1}}{N} & -\frac{\beta_{2} x_{1}}{N} & 0 & -\beta_{3} x_{1} \\
0 & \kappa_{1} & \kappa_{2} & \kappa_{3} & 0
\end{array}\right]
\end{gathered}
$$

and

$$
A_{2}(Z)=\left[\begin{array}{ccccc}
-a & \frac{\beta_{1} x_{1}}{N} & \frac{\beta_{2} x_{1}}{N} & 0 & \beta_{3} x_{1} \\
\alpha_{1} & -b & 0 & 0 & 0 \\
0 & \alpha_{2} & -c & 0 & 0 \\
0 & \gamma_{1} & \gamma_{2} & -d & 0 \\
0 & \frac{\alpha_{3}}{N} & \frac{\alpha_{3}}{N} & 0 & -\mu_{1}
\end{array}\right]
$$

where $d=\left(\delta_{2}+\kappa_{3}\right)$. We want to show that the five sufficient conditions of Kamgang-Sallet Theorem in [10] are satisfied as follows:

(i) The system (4) - (11) is a dynamical system on $\Omega$, as defined and shown in section $V$. (ii) The eigenvalues of $A_{1}(Z)$ are real and negative, thus the system $\dot{Z}_{1}=A_{1}(Z)\left(Z_{1}-Z_{1}^{*}\right)+$ $A_{12}(Z) Z_{2}$ is globally asymptotically stable at the equilibrium $Z_{1}^{*}$.

(iii) The matrix $A_{2}(Z)$ is a Metzler matrix, i.e. a matrix such that off diagonal terms are non negative and is irreducible for any given $Z \in$ $\Omega$.

(iv) There exists a matrix $\overline{A_{2}}$, which is an upper bound for the set $M=A_{2}(Z): Z \in \Omega$. Indeed,

$$
\bar{A}_{2}=\left[\begin{array}{ccccc}
-a & \beta_{1} & \beta_{2} & 0 & \beta_{3} x_{1}^{*} \\
\alpha_{1} & -b & 0 & 0 & 0 \\
0 & \alpha_{2} & -c & 0 & 0 \\
0 & \gamma_{1} & \gamma_{2} & -d & 0 \\
0 & \frac{\alpha_{3}}{N^{*}} & \frac{\alpha_{3}}{N^{*}} & 0 & -\mu_{1}
\end{array}\right]
$$

is an upper bound for $M$.

(v) For $R_{0}<1, \lambda$ is the eigenvalue of $\overline{A_{2}}$, $\alpha\left(\overline{A_{2}}\right)=\max \{\operatorname{Re}(\lambda): \lambda\} \leq 0$

To check condition (v) we will use the following lemma which is a characterization of Metzler stable matrices:

Lemma 4. Let $M$ be a square matrix written in block form

$$
D=\left[\begin{array}{ll}
A & B \\
C & D
\end{array}\right]
$$

with $A$ and $D$ being square matrices. $M$ is Metzler stable if and only if matrices $A$ and $D-C A^{-1} B$ are Metzler stable.

Using Lemma 4, matrix $\overline{A_{2}}$ can be expressed in the form of matrix $\mathrm{M}$ with:

$$
\begin{aligned}
& A=\left[\begin{array}{ccc}
-a & \beta_{1} & \beta_{2} \\
\alpha_{1} & -b & 0 \\
0 & \alpha_{2} & -c
\end{array}\right], \quad B=\left[\begin{array}{cc}
0 & \beta_{3} x_{1}^{*} \\
0 & 0 \\
0 & 0
\end{array}\right] \\
& C=\left[\begin{array}{ccc}
0 & \gamma_{1} & \gamma_{2} \\
0 & \frac{\alpha_{3}}{N^{*}} & \frac{\alpha_{3}}{N^{*}}
\end{array}\right], \quad D=\left[\begin{array}{cc}
-d & 0 \\
0 & -\mu_{1}
\end{array}\right] .
\end{aligned}
$$

Clearly A, is a stable Metzler matrix and after computations we obtain $D-C A^{-1} B$ is a stable 
M H Machingauta, B Lungu, E M Lungu, COVID-19 changing the face of the world. Can sub-Sahara ...

Metzler matrix if and only if

$$
\begin{aligned}
R_{0}^{\max }= & \frac{\alpha_{1} \beta_{1}}{a b}+\frac{\alpha_{1} \alpha_{2} \beta_{2}}{a b c} \\
& +\frac{\alpha_{1} \alpha_{3} \beta_{3}\left(c+\alpha_{2}\right)}{a b c \mu_{1}} \\
\leq & 1 .
\end{aligned}
$$

\section{Endemic Equilibrium Point (EEP)}

Solving for the system of equations (4) (11) by equating the RHS to zero, we find the coordinates of the EEP given by $\zeta_{1}=$ $\left(x_{1}^{*}, x_{2}^{*}, x_{3}^{*}, x_{4}^{*}, x_{5}^{*}, x_{6}^{*}, x_{7}^{*}\right)$, where

$$
\begin{aligned}
& x_{1}^{*}=\frac{a b c N^{*} \mu_{1}}{\alpha_{1} a_{33}} \\
& x_{2}^{*}=\frac{a_{22}\left(R_{0}-1\right)}{a \alpha_{1} a_{33}} \\
& x_{3}^{*}=\frac{a_{22}\left(R_{0}-1\right)}{a b a_{33}} \\
& x_{4}^{*}=\frac{\alpha_{2} a_{22}\left(R_{0}-1\right)}{a b c a_{33}} \\
& x_{5}^{*}=\frac{a_{22} a_{44}\left(R_{0}-1\right)}{a b c d a_{33}} \\
& x_{6}^{*}=\frac{\kappa_{3} a_{22} a_{44} a_{55}\left(R_{0}-1\right)}{a b c d \mu a_{33}} \\
& x_{7}^{*}=\frac{\alpha_{3}\left(\alpha_{2}+c\right) a_{22}\left(R_{0}-1\right)}{a b c N^{*} \mu_{1} a_{33}}
\end{aligned}
$$

and

$$
\begin{aligned}
& a_{22}=a b c N^{*} \mu \mu_{1} \\
& a_{33}=\left(c+\alpha_{2}\right) \alpha_{3} \beta_{3}+\left(c \beta_{1}+\alpha_{2} \beta_{2}\right) \mu_{1} \\
& a_{44}=\left(\alpha_{2} \gamma_{2}+c \gamma_{1}\right) \\
& a_{55}=\left(c d \kappa_{1}+d \kappa_{2} \alpha_{2}\right) .
\end{aligned}
$$

The coordinates $(13)-(18)$ exist if and only if $R_{0}>1$.

\section{1) Global stability of the Endemic Equilibrium} Point: The global stability of the EEP is explored by proving the following theorem:

Theorem 5. If $R_{0}>1$ then the EEP given by $\zeta_{1}$ is globally asymptotically stable in the region $\Omega$.
Proof. Following the work of [5], we construct a Lyapunov function $L$ of the type:

$$
L\left(x_{i}\right)=\sum_{i=1}^{7}\left(x_{i}-x_{i}^{*}-x_{i}^{*} \ln \frac{x_{i}}{x_{i}^{*}}\right) .
$$

Differentiating $L$ with respect to $x_{i}$ gives:

$$
\frac{d L}{d t}=\sum_{i=1}^{7}\left(\frac{x_{i}-x_{i}^{*}}{x_{i}}\right) \frac{d x_{i}}{d t}
$$

Substituting for $\frac{d x_{i}}{d t}, i=1, \ldots, 7$, we get

$$
\begin{aligned}
\frac{d L}{d t}= & \left(\frac{x_{1}-x_{1}^{*}}{x_{1}}\right) \\
& {\left[\pi-\frac{\left(\beta_{1} x_{3}+\beta_{2} x_{4}\right) x_{1}}{N}-\beta_{3} x_{7} x_{1}-\mu x_{1}\right] } \\
& +\left(\frac{x_{2}-x_{2}^{*}}{x_{2}}\right) \\
& {\left[\frac{\left(\beta_{1} x_{3}+\beta_{2} x_{4}\right) x_{1}}{N}+\beta_{3} x_{7} x_{1}-a x_{2}\right] } \\
& +\left(\frac{x_{3}-x_{3}^{*}}{x_{3}}\right)\left[\alpha_{1} x_{2}-b x_{3}\right] \\
& +\left(\frac{x_{4}-x_{4}^{*}}{x_{4}}\right)\left[\alpha_{2} x_{3}-c x_{4}\right] \\
& +\left(\frac{x_{5}-x_{5}^{*}}{x_{5}}\right)\left[\gamma_{1} x_{3}+\gamma_{2} x_{4}-d x_{5}\right] \\
& +\left(\frac{x_{6}-x_{6}^{*}}{x_{6}}\right) \\
& {\left[\kappa_{1} x_{3}+\kappa_{2} x_{4}+\kappa_{3} x_{5}-\mu x_{6}\right] } \\
& +\left(\frac{x_{7}-x_{7}^{*}}{x_{7}}\right)\left[\frac{\alpha_{3}\left(x_{3}+x_{4}\right)}{N}-\mu_{1} x_{7}\right] \\
= & A_{33}-A_{22},
\end{aligned}
$$


M H Machingauta, B Lungu, E M Lungu, COVID-19 changing the face of the world. Can sub-Sahara ...

where

$$
\begin{aligned}
A_{22}= & \left(\frac{x_{1}-x_{1}^{*}}{x_{1}}\right)^{2}\left[\frac{\left(\beta_{1} x_{3}+\beta_{2} x_{4}\right)}{N}+\beta_{3} x_{7}+\mu\right] \\
& +\left[\frac{x_{1}^{*}}{x_{1}} \pi+\frac{\left(\beta_{1} x_{3}+\beta_{2} x_{4}\right) x_{1}^{*}}{N}+\beta_{3} x_{7} x_{1}^{*}+\mu x_{1}^{*}\right] \\
& +\left(\frac{x_{2}-x_{2}^{*}}{x_{2}}\right)^{2} a+a x_{2}^{*} \\
& +\frac{x_{2}^{*}}{x_{2}}\left[\frac{\left.\beta_{1} x_{3}+\beta_{2} x_{4}\right) x_{1}}{N}+\beta_{3} x_{7} x_{1}\right] \\
& +\left(\frac{x_{3}-x_{3}^{*}}{x_{3}}\right)^{2} b+b x_{3}^{*}+\frac{x_{3}^{*}}{x_{3}} \alpha_{1} x_{2} \\
& +\left(\frac{x_{4}-x_{4}^{*}}{x_{4}}\right)^{2} c+c x_{4}^{*}+\frac{x_{4}^{*}}{x_{4}} \alpha_{2} x_{3} \\
& +\left(\frac{x_{5}-x_{5}^{*}}{x_{5}}\right)^{2} d+d x_{5}^{*}+\frac{x_{5}^{*}}{x_{5}}\left(\gamma_{1} x_{3}+\gamma_{2} x_{4}\right) \\
& +\left(\frac{x_{6}-x_{6}^{*}}{x_{6}}\right)^{2} \mu+\mu x_{6}^{*} \\
& +\frac{x_{6}^{*}}{x_{6}}\left(\kappa_{1} x_{3}+\kappa_{2} x_{4}+\kappa_{3} x_{5}\right) \\
& +\left(\frac{x_{7}-x_{7}^{*}}{x_{7}}\right)^{2} \mu_{1}+\mu_{1} x_{7}^{*}+\frac{x_{7}^{*}}{x_{7}} \frac{\alpha_{3}\left(x_{3}+x_{4}\right)}{N}
\end{aligned}
$$

and

\begin{tabular}{|c|c|c|}
\hline Parameter & Value/range & Source \\
\hline$\beta_{1}$ & 0.492 & {$[8]$} \\
\hline$\beta_{2}$ & 1.30 & [20] \\
\hline$\beta_{3}$ & 0 & estimate \\
\hline$\delta_{1}$ & 0.015 & [19] \\
\hline$\delta_{2}$ & $\frac{4826}{52578}$ & [33] \\
\hline \multirow{3}{*}{$\alpha_{1}$} & & \\
\hline & 1 & \multirow{2}{*}{ [20] } \\
\hline & 3.21 & \\
\hline \multirow{2}{*}{$\alpha_{2}$} & 1 & \multirow{2}{*}{ [20] } \\
\hline & $\overline{2.27}$ & \\
\hline$\alpha_{3}$ & 0 & estimate \\
\hline$\gamma_{1}$ & {$[0,0.6]$} & estimate \\
\hline$\gamma_{2}$ & {$[0,0.6]$} & estimate \\
\hline$\kappa_{1}$ & $\overline{6}$ & [8] \\
\hline \multirow[b]{2}{*}{$\kappa_{2}$} & 1 & \multirow{2}{*}{ [8] } \\
\hline & 10 & \\
\hline$\kappa_{3}$ & 1 & [8] \\
\hline$\mu$ & $\begin{array}{c}14 \\
0.00003032\end{array}$ & [35] \\
\hline$\mu_{1}$ & 0 & estimate \\
\hline$\pi$ & $\mu \times 400000$ & [11] \\
\hline
\end{tabular}

$$
\begin{aligned}
A_{33}= & \pi+\frac{x_{1}^{*^{2}}}{x_{1}}\left[\frac{\left(\beta_{1} x_{3}+\beta_{2} x_{4}\right)}{N}+\beta_{3} x_{7}+\mu\right] \\
& +\left[\frac{\left(\beta_{1} x_{3}+\beta_{2} x_{4}\right)}{N}+\beta_{3} x_{7}+\mu\right] \\
& +\frac{x_{2}^{*^{2}}}{x_{2}} a+\alpha_{1} x_{2}+\frac{x_{3}^{*^{2}}}{x_{3}} b+\alpha_{2} x_{3}+\frac{x_{4}^{*^{2}}}{x_{4}} c \\
& +\gamma_{1} x_{3}+\gamma_{2} x_{4}+\frac{x_{5}^{*^{2}}}{x_{5}} d+\kappa_{1} x_{3}+\kappa_{2} x_{4} \\
& +\kappa_{3} x_{5}+\frac{x_{6}^{*^{2}}}{x_{6}} \mu+\frac{\alpha_{3}\left(x_{3}+x_{4}\right)}{N}+\frac{x_{7}^{*^{2}}}{x_{7}} \mu_{1} .
\end{aligned}
$$

Since all the parameters used in the system (4) (11) are non negative we have $\frac{d L}{d t} \leq 0$ for $A_{33} \leq$ $A_{22}$ and $\frac{d L}{d t}=0$ if and only if $x_{1}=x_{1}^{*}, x_{2}=$ $x_{2}^{*}, x_{3}=x_{3}^{*}, x_{4}=x_{4}^{*}, x_{5}=x_{5}^{*}, x_{6}=x_{6}^{*}, x_{7}=x_{7}^{*}$. Thus by La-Salle's invariance principle, the EEP is globally asymptotically stable.
TABLE I: Numerical values for the parameters of the Italian case

\section{Numerical Simulations}

We want to find optimal isolation rates $\gamma_{1}$ and $\gamma_{2}$, using Matlab programs, under which the number of infected individuals will not overwhelm the hospital capacity, $H$. We performed numerical simulations using data from the Italian coronavirus epidemic from $31^{\text {st }}$ January to the $15^{\text {th }}$ of May 2020 to demonstrate that our model can accurately reproduce the recorded data on numbers of infected and dead individuals. The parameter values used for numerical simulations are given in Table $I$.

1) Sensitivity Analysis: Five parameters, $\beta_{3}$, $\alpha_{3}, \gamma_{1}, \gamma_{2}$ and $\mu_{1}$ in Table $I$ have been estimated based on the number of hospitalized cases in the Lombardy region of Northern Italy. We have analyzed how sensitive the reproduction number is to the changes in these five parameters (Figure 


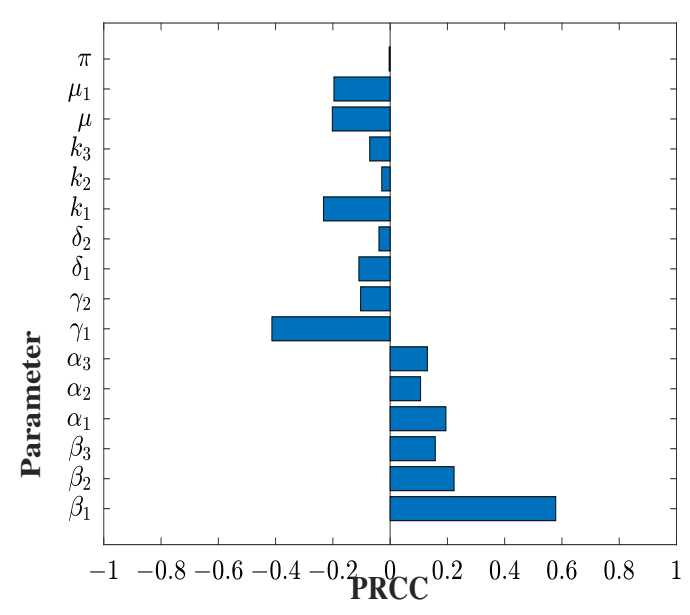

Fig. 5: A diagram showing the sensitivity of $R_{0}$ to various model parameters.

5). The parameters $\alpha_{3}$ and $\beta_{3}$ are important and provide insight into understanding how the subSahara Africa situation will differ from the Italian or in general the situation in Western countries, where we assumed $\alpha_{3}=0$.

\section{A. Italy}

Lombardy is a region with a population of approximately 10 million. By an iterative procedure, since we know the number of people infected with the coronavirus from $31^{\text {st }}$ January to $22^{\text {nd }}$ March 2020 , we have estimated that the number of people susceptible to infection by the virus, through their failure to observe prevention measures such as self quarantine, social distancing etc at the start of the coronavirus epidemic, was about 400000 . As at $31^{\text {st }}$ January 2020, the number who were potentially exposed to the infection is estimated to be around 200 individuals. On the $31^{\text {st }}$ January 2020, Italy recorded its first 3 cases of individuals infected with the coronavirus. Based on this initial data, we present examples of strategies by fixing the rate of isolating symptomatic infectives and then finding the corresponding rate of isolating asymptomatic infectives which ensures that the combined number of infectives does not exceed the hospital bed capacity and vice versa. The examples discussed here are not unique but are typical of other scenarios and provide insight into the following:

(i) how the infection curve can be flattened to ensure that the hospital bed capacity is not exceeded.

(ii) For non optimal cases, where hospital bed capacity is exceeded, to quantify the number of infectives for each non optimal case which must be safely isolated outside hospital facilities.

According to [23], Italy has 12.5 beds per 100000 individuals of Intensive Care Unit (ICU) or critical care beds (CCB) beds and 3.18 beds per 1000 individuals ordinary hospital beds. For Lombardy, this data (combining both ordinary and ICU hospital beds) gives 33182 beds.

Figure 6 presents an example for a fixed rate $\gamma_{2}=0.33$ of isolating symptomatic infectives. We find that the optimal rate of securing asymptomatic infectives when there is a lockdown, $\pi=0$, should be $\gamma_{1}=0.46$. Converting these rates to time, we see that asymptomatic infectives should be identified, hospitalized in a time almost 1.5 times faster than the time of hospitalizing symptomatic infectives. Currently, every nation is experiencing a lack of materials and equipment to conduct tests. This rate of hospitalizing asymptomatic infectives would be difficult to achieve as there has been a shortage of testing materials, and most governments have decided they would only test individuals who present with COVID-19 symptoms. Moreover, the time it is taking to obtain test results $2-3$ days is slowing down the testing significantly.

For this strategy, we have determined the number of infected people that should be safely secured for varying values of $\gamma_{1}$ at peak infection. For $\gamma_{1}=0$, the number in excess of hospital bed capacity that must be secured is 22408 , for $\gamma_{1}=0.3$, the number in excess of hospital bed capacity that must be secured is 13407 . For the non optimal cases, if the hospital overflow bed capacity is increased by $50 \%$ (as was done in most Western countries) the only case that would have accommo- 


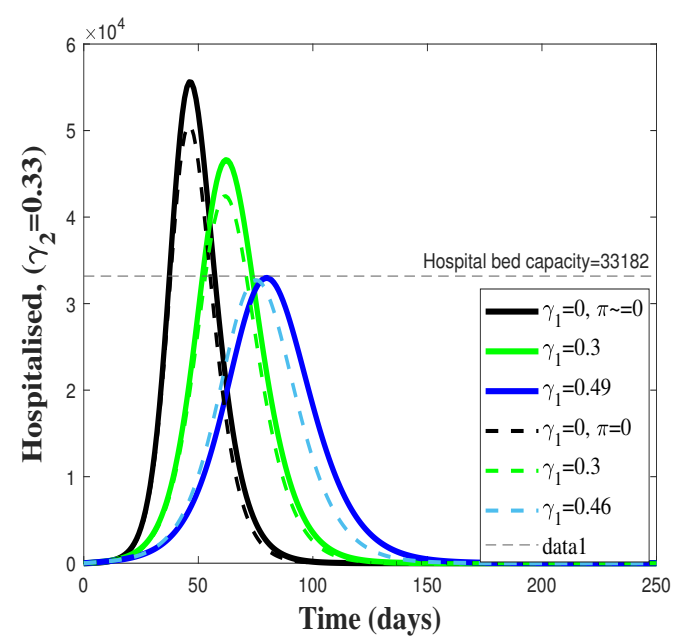

Fig. 6: Population level plots for hospitalized individuals for varying values of $\gamma_{1}$

dated the overflow is $\left(\gamma_{1}, \gamma_{2}\right)=(0.3,0.33)$. Figure 6 shows that when inter zonal movement is allowed, $\pi \neq 0$, the number of people needing hospitalization or to be safely isolated increases. The optimum removal rates with inter-zonal movement are given by $\left(\gamma_{1}^{*}, \gamma_{2}^{*}\right)=(0.49,0.33)$, implying that asymptomatic infectives must be isolated in a time 1.6 times faster than when there is no inter zonal movement. The optimal solutions in Figure 6 show that the peak hospitalization capacity occurs earlier when there is a lockdown $(\pi=0)$ than when there is inter-zonal movement $(\pi \neq 0)$.

Figure 7 presents a strategy where the rate of hospitalizing symptomatic infectives is fixed at $\gamma_{2}=0.6$. The optimal rate for $\gamma_{1}$ which flattens the hospital admission curve is found to be $\gamma_{1}=0.16$. In other words, almost one third of the effort must be devoted to testing, contact tracing and hospitalizing asymptomatic infectives.

For this strategy the situation regarding the non optimal cases is as follows: for $\gamma_{1}=0$ the number needed to be secured is 10097 , which is too high to accommodate.

Using optimization techniques to find $\gamma_{1}^{*}$ and $\gamma_{2}^{*}$ we have found and demonstrated in Figure 8 that the optimum rates are $\left(\gamma_{1}^{*}, \gamma_{2}^{*}\right)=(0.6,0.26)$. This optimum pair implies that the effort devoted to

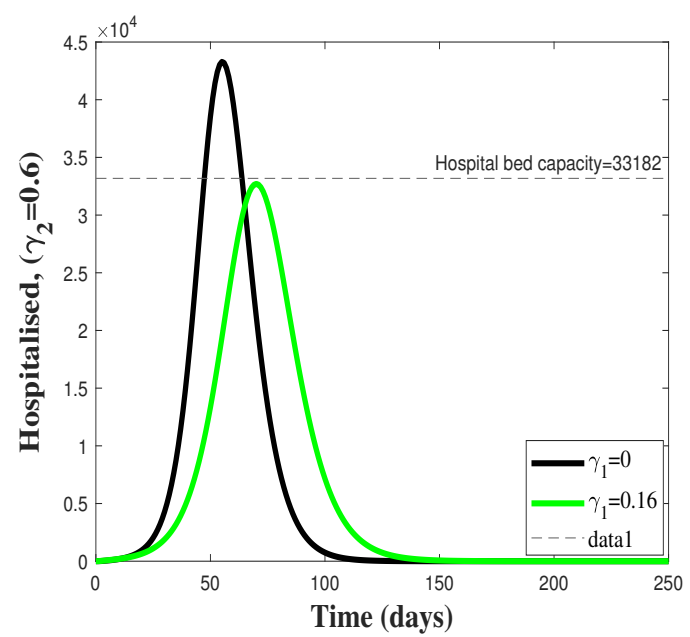

Fig. 7: Population level plots for hospitalized individuals for varying values of $\gamma_{1}$

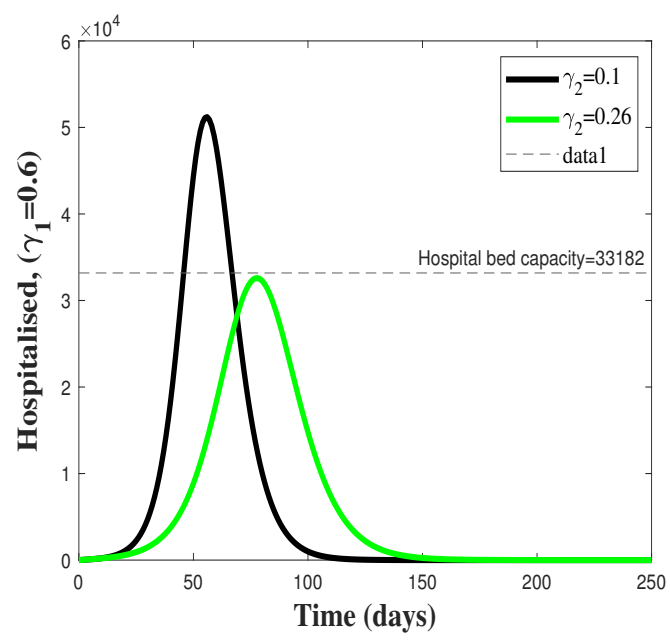

Fig. 8: Population level plots for hospitalized individuals for varying values of $\gamma_{2}$

testing, contact tracing and isolating symptomatic infectives must be 2.3 times higher than that of isolating asymptomatic infectives.

Figure 9 shows that for $\gamma_{1}=0.3$ the optimum pair is $\left(\gamma_{1}^{*}, \gamma_{2}^{*}\right)=(0.3,0.45)$. This strategy targets to remove symptomatic infectives faster than the asymptomatic infectives. This strategy removes symptomatic infectives in a time at least one and half times faster than the time of isolating 
M H Machingauta, B Lungu, E M Lungu, COVID-19 changing the face of the world. Can sub-Sahara ...

TABLE II: Comparison of deaths at different optimal cases

\begin{tabular}{|c|c|c|c|c|c|c|c|}
\hline Strategy & $\gamma_{1}$ & $\gamma_{2}$ & $\begin{array}{c}30 \text { days } \\
\delta=0.01\end{array}$ & $\begin{array}{c}60 \text { days } \\
\delta=0.03\end{array}$ & $\begin{array}{c}90 \text { days } \\
\delta=0.02\end{array}$ & $\begin{array}{c}105 \text { days } \\
\delta=0.015 \\
\text { Lockdown }\end{array}$ & $\begin{array}{c}105 \text { days } \\
\delta=0.09 \\
\text { No lockdown }\end{array}$ \\
\hline 1 & 0.49 & 0.33 & $\begin{array}{c}210 \\
(29)\end{array}$ & $\begin{array}{c}10126 \\
(11591)\end{array}$ & $\begin{array}{c}32108 \\
(27682)\end{array}$ & $\begin{array}{c}31433 \\
(31368)\end{array}$ & 120200 \\
\hline 2 & 0.16 & 0.6 & $\begin{array}{c}226 \\
(29)\end{array}$ & $\begin{array}{c}12861 \\
(11591)\end{array}$ & $\begin{array}{c}33994 \\
(27682)\end{array}$ & $\begin{array}{c}31202 \\
(31368)\end{array}$ & 115690 \\
\hline 3 & 0.6 & 0.26 & $\begin{array}{c}200 \\
(29)\end{array}$ & $\begin{array}{c}8989 \\
(11591)\end{array}$ & $\begin{array}{c}30734 \\
(27682)\end{array}$ & $\begin{array}{c}31162 \\
(31368)\end{array}$ & 120640 \\
\hline 4 & 0.3 & 0.45 & $\begin{array}{c}217 \\
(29)\end{array}$ & $\begin{array}{c}11179 \\
(11591)\end{array}$ & $\begin{array}{c}32771 \\
(27682)\end{array}$ & $\begin{array}{c}31135 \\
(31368)\end{array}$ & 117600 \\
\hline
\end{tabular}

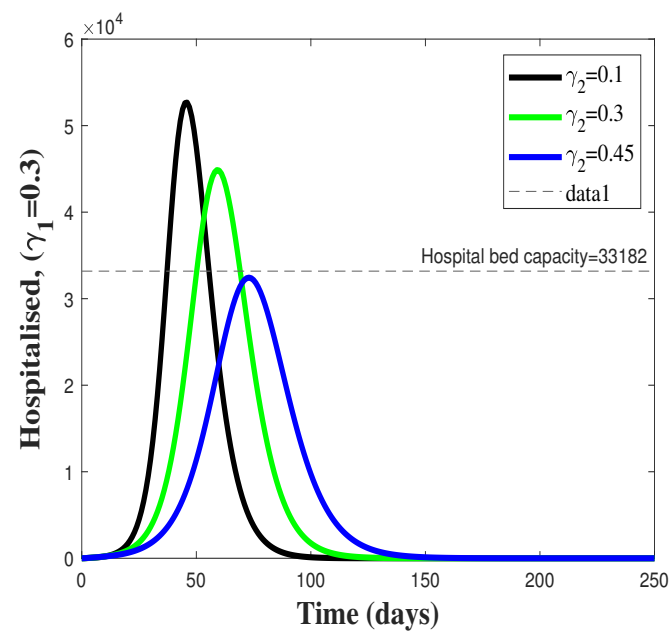

Fig. 9: Population level plots for hospitalized individuals for varying values of $\gamma_{2}$

asymptomatic infectives.

To illustrate the impact of non adherence to prevention measures, such as social distancing, not wearing masks etc, on the optimal case given in Figure 9 , we considered how the case $\beta_{3} \neq 0$ for Italy would have altered the conclusions presented in Figures 6 to 9. Figure 10 shows that if we vary the parameter $\alpha_{3}$ the hospital bed capacity for Lombardy in Italy would have been exceeded for any $\alpha_{3} \geq 0.01$. This suggests that high standards of hygiene are key to controlling COVID-19 infections.

We can see from the examples of the four strategies illustrated in Figures 6, 7, 8, 9 that there is no unique way of flattening the curve in order to

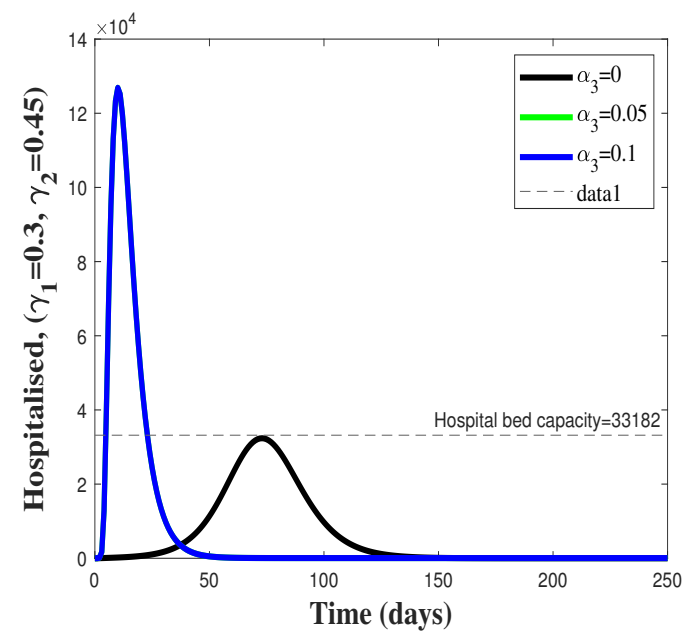

Fig. 10: Population level plots for hospitalized individuals for varying values of $\alpha_{3}$

protect the hospital bed capacity. The question we address is how does one choose the best strategy among the optimal strategies? Table $I I$ gives the number of deaths resulting from the four strategies above and compares each strategy with the actual number of recorded deaths. We conclude that the best strategy is one which reduces the number of deaths. In this case (Table II) any strategy that isolates the symptomatic infectives faster is preferable as it is more economical.

\section{B. Optimal Control}

1) Optimal control without incorporating household and community exposure: To study measures that reduce disease transmission, 
such as lockdown or isolation of infectives, we introduced two controls, $u_{1}$ and $u_{2}$, on the infection coefficients $\beta_{1}$ and $\beta_{2}$ as shown below:

$$
\begin{aligned}
\frac{d x_{1}}{d t}= & \pi-\left(1-u_{1}\right) \frac{\beta_{1} x_{3} x_{1}}{N}-\left(1-u_{2}\right) \frac{\beta_{2} x_{4} x_{1}}{N} \\
& -\beta_{3} x_{7} x_{1}-\mu x_{1} \\
\frac{d x_{2}}{d t}= & \left(1-u_{1}\right) \frac{\beta_{1} x_{3} x_{1}}{N}+\left(1-u_{2}\right) \frac{\beta_{2} x_{4} x_{1}}{N} \\
& +\beta_{3} x_{7} x_{1}-\left(\alpha_{1}+\mu\right) x_{2} \\
\frac{d x_{3}}{d t}= & \alpha_{1} x_{2}-\left(\gamma_{1}+\alpha_{2}+\mu+\kappa_{1}\right) x_{3} \\
\frac{d x_{4}}{d t}= & \alpha_{2} x_{3}-\left(\gamma_{2}+\delta_{1}+\kappa_{2}\right) x_{4} \\
\frac{d x_{5}}{d t}= & \gamma_{1} x_{3}+\gamma_{2} x_{4}-\left(\delta_{2}+\kappa_{3}\right) x_{5} \\
\frac{d x_{6}}{d t}= & \kappa_{1} x_{3}+\kappa_{2} x_{4}+\kappa_{3} x_{5}-\mu x_{6}
\end{aligned}
$$

and

$$
\frac{d x_{7}}{d t}=\frac{\alpha_{3}\left(x_{3}+x_{4}\right)}{N}-\mu_{1} x_{7}
$$

We want to minimize the objective functional given by

$$
\begin{aligned}
J\left(u_{1}, u_{2}\right)= & \int_{0}^{t_{T}} A_{1} x_{2}+A_{2} x_{3}+A_{3} x_{4} \\
& +\frac{1}{2} A_{4} u_{1}^{2}+\frac{1}{2} A_{5} u_{2}^{2} .
\end{aligned}
$$

The goal is to find a set of controls that minimize the number of susceptible individuals who come into contact with infected individuals. Let $u_{1}^{*}, u_{2}^{*}$ be the optimal controls. The problem is to find

$$
J\left(u_{1}^{*}, u_{2}^{*}\right)=\min J\left(u_{1}, u_{2}\right),\left(u_{1}, u_{2}\right) \in U,
$$

subject to system $(19)-(25)$, where $U$ is the set of measure functions defined from $\left[0, t_{T}\right]$ to $[0,1]$.

The optimality conditions are given by,

$$
\begin{aligned}
& u_{1}^{*}=\min \left\{\max \left[0, D_{1}\left(\frac{\beta_{1} x_{3} x_{1}}{A_{4} N}\right)\right], u_{1} \max \right\}, \\
& u_{2}^{*}=\min \left\{\max \left[0, D_{1}\left(\frac{\beta_{2} x_{4} x_{1}}{A_{5} N}\right)\right], v_{2} \max \right\},
\end{aligned}
$$

where $D_{1}=\left(\lambda_{x_{2}}-\lambda_{x_{1}}\right)$.

Calculation for $u_{1}^{*}$ and $u_{2}^{*}$ is based on Pontryagin's Maximum Principle (see [14]) for a detailed description.
2) Optimal control incorporating household and community exposure.: To study the impact that household and community exposure has on disease transmission control measures, we modify equations (19) $-(25)$ by adding an additional term $\lambda$ given in (3) which captures household and community exposure of an individual. The modified equations are given by:

$$
\begin{aligned}
\frac{d x_{1}}{d t}= & \pi-\left(1-u_{1}+\lambda\right) \frac{\beta_{1} x_{3} x_{1}}{N} \\
& -\left(1-u_{2}+\lambda\right) \frac{\beta_{2} x_{4} x_{1}}{N}-\beta_{3} x_{7} x_{1} \\
& -\mu x_{1} \\
\frac{d x_{2}}{d t}= & \left(1-u_{1}+\lambda\right) \frac{\beta_{1} x_{3} x_{1}}{N} \\
& +\left(1-u_{2}+\lambda\right) \frac{\beta_{2} x_{4} x_{1}}{N}+\beta_{3} x_{7} x_{1} \\
& -\left(\alpha_{1}+\mu\right) x_{2} \\
\frac{d x_{3}}{d t}= & \alpha_{1} x_{2}-\left(\gamma_{1}+\alpha_{2}+\mu+\kappa_{1}\right) x_{3} \\
\frac{d x_{4}}{d t=} & \alpha_{2} x_{3}-\left(\gamma_{2}+\delta_{1}+\kappa_{2}\right) x_{4} \\
\frac{d x_{5}}{d t}= & \gamma_{1} x_{3}+\gamma_{2} x_{4}-\left(\delta_{2}+\kappa_{3}\right) x_{5} \\
\frac{d x_{6}}{d t}= & \kappa_{1} x_{3}+\kappa_{2} x_{4}+\kappa_{3} x_{5}-\mu x_{6}
\end{aligned}
$$

and

$$
\frac{d x_{7}}{d t}=\frac{\alpha_{3}\left(x_{3}+x_{4}\right)}{N}-\mu_{1} x_{7}
$$

where $\lambda$ is given by $(3)$.

The optimality conditions that we want to satisfy are the same as those for equations $(19)-(25)$. We consider the non optimal strategy in Figure 6 using equations $(19)-(25)$ and equations (28) (34) for $\gamma_{1}=0.3$ and $\gamma_{2}=0.33$ where $u_{1}=0$ and $u_{2}=0$. If we use the controls $u_{1}^{*} \geq 0.2, u_{2}^{*} \geq$ 0.2 and $\left(\gamma_{1}, \gamma_{2}\right)=(0.3,0.33)$ we obtain Figures $11(a)-11(e)$. It is clear from Figure $11(e)$ that household and community disease transmission would increase the number hospitalized though the numbers would still be below the hospital bed capacity for optimal values of $\gamma_{1}$ and $\gamma_{2}$. The number of susceptible individuals (Figure 11(a)) would decline due to increased infection rates. 

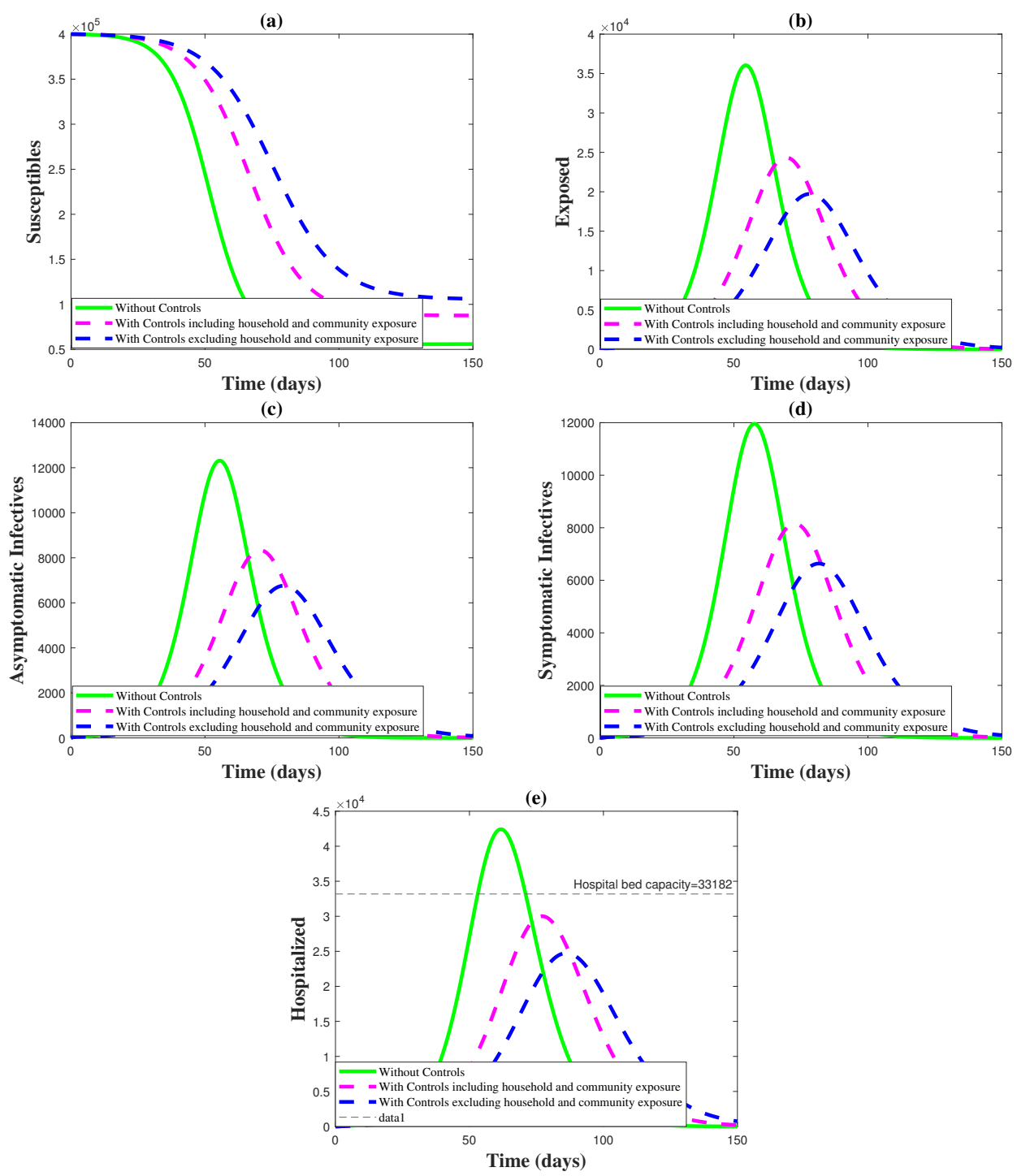

Fig. 11: A comparison of state variables with and without controls

C. Sub-Sahara Africa: South Africa as an example.

To provide insight into the sub-Sahara Africa outlook, simulations were done using data from South Africa, one of the countries in this region with the highest number of coronavirus cases and with the best medical facilities. It is our view that if South Africa cannot cope then most, if not all, countries in sub-Sahara Africa would not cope. On the $4^{\text {th }}$ of March 2020, South Africa recorded its first case of the coronavirus. As of the $1^{\text {st }}$ of April 2020 the highest case counts of coronavirus had been reported in 3 provinces namely Gauteng, KwaZulu Natal and the Western Cape. The 3 provinces had 1380 reported coronavirus cases distributed as follows: 645 in Gauteng, 326 in KwaZulu Natal and 186 in the Western Cape. These 3 provinces have a total population of 33309473 people. The number of people infected with the coronavirus from the $4^{\text {th }}$ of March to the $1^{\text {st }}$ April 2020 is known, we have estimated that the number of people susceptible to infection by 
M H Machingauta, B Lungu, E M Lungu, COVID-19 changing the face of the world. Can sub-Sahara ...

TABLE III: Numerical values for the parameters of the South African case

\begin{tabular}{|c|c|c|}
\hline Parameter & Value/range & Source \\
\hline$\beta_{1}$ & 0.492 & [8] \\
\hline$\beta_{2}$ & 1.30 & [20] \\
\hline$\beta_{3}$ & 0.001006082 & [11] \\
\hline$\delta_{1}$ & 0.015 & [19] \\
\hline$\delta_{2}$ & $\frac{5}{1380}$ & [33] \\
\hline$\alpha_{1}$ & 1 & 20 \\
\hline & $\overline{3.21}$ & \\
\hline$\alpha_{2}$ & 1 & [20] \\
\hline$\alpha_{3}$ & $5.3346 \times 10^{-6}$ & estimate \\
\hline$\gamma_{1}$ & {$[0,0.77]$} & estimate \\
\hline$\gamma_{2}$ & {$[0,0.33]$} & estimate \\
\hline$\kappa_{1}$ & $\frac{1}{6}$ & 8] \\
\hline & 1 & \\
\hline$\kappa_{2}$ & $\overline{10}$ & [8] \\
\hline$\kappa_{3}$ & $\frac{1}{14}$ & [8] \\
\hline$\mu$ & 0.00004290 & [36] \\
\hline$\mu_{1}$ & 0.00274 & [11] \\
\hline$\pi$ & $\mu \times 1200000$ & [11] \\
\hline
\end{tabular}

the virus through failure to self quarantine, self isolate, observe social distancing etc was about 1200000 . At that time the number who were potentially exposed to the infection is estimated to be about 1000 individuals.

Except for $\beta_{3}, \pi, \alpha_{3}, \mu, \mu_{1}$ and $\delta_{2}$, we use the parameters in Table 1 for Italy. This is justified on the basis that Italian family bonds are similar to sub-Sahara Africa. The values used for this simulation are given in Table 3. According to [4], Gauteng province, KwaZulu Natal Province and the Western Cape province combined have a hospital bed capacity of 62787 . This number includes both ICU and ordinary hospital beds.

Figure 12 compares real time epidemic curves for Italy, Spain, the United Kingdom and South Africa for the first 40 days of the epidemic. Each country implemented the lockdown strategy at different stages of infection. Italy introduced the lockdown on the $9^{\text {th }}$ of March 2020 when the total number of individuals infected with COVID-
19 was 9172 . Spain introduced the lockdown on the $15^{\text {th }}$ of March 2020 when the total number of those infected was 7798 . The United Kingdom introduced the lockdown on the $23^{\text {rd }}$ of March 2020 when the number of individuals infected was 6650. South Africa introduced the lockdown on the $27^{\text {th }}$ of March 2020 when the number infected was only 1170 . Figure 12 shows different infection trends for the four countries.

It is obvious that South Africa which introduced the lockdown early enough displays an epidemic which rises at a gentle rate. We consider the problem of finding the hospitalization rates $\gamma_{1}$ and $\gamma_{2}$ for which the hospital bed capacity of 62787 would suffice.

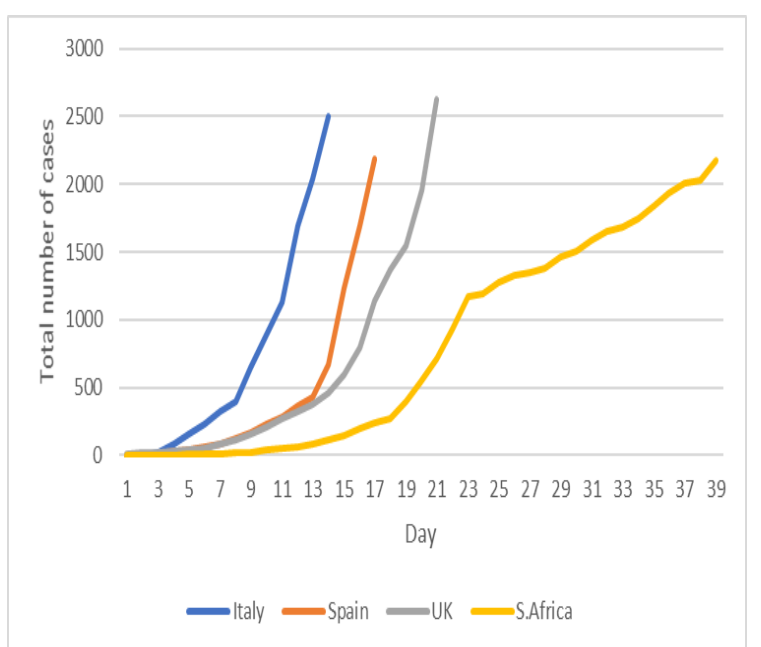

Fig. 12: Total covid cases, UK, Spain, Italy, South Africa

1) Scenario with no lockdown measures: Figure 13 shows that for a fixed rate $\gamma_{2}=0.33$ of symptomatic infectives, the least rate of isolating asymptomatic infectives should be $\gamma_{1}=0.77$ for $\phi=0$ (implying that the population must observe all the prevention measures ,social distancing, mask wearing etc) but that for $\phi \neq 0$ the hospital bed capacity is never sufficient. 


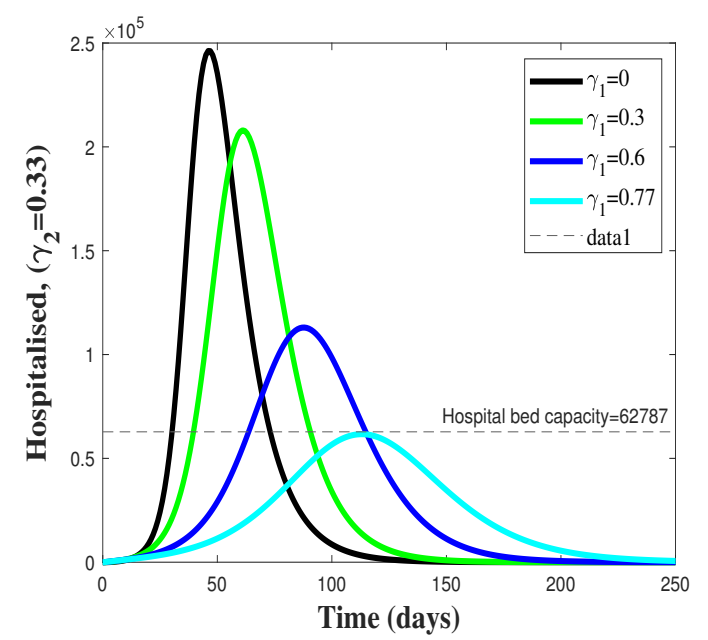

Fig. 13: Population level plots for hospitalized individuals for varying values of $\gamma_{1}$

For the strategies in Figure 13, the numbers of people that must be isolated are given in Table 4 .

Table 4 presents cases when the hospital bed capacity, denoted by $H$, is increased by $50 \%$ as was done in Italy, Spain and the State of New York, USA. The overflow is given by $\left(H_{P}-H \times 1.5\right)$. We can see that no pair of isolating infectives $\gamma_{1}$ and $\gamma_{2}$ would accommodate the number of infected individuals in the 3 provinces of South Africa within hospital facilities. The overflow capacity would be too large for any non-optimal pair $\gamma_{1}$ and $\gamma_{2}$ and $\phi \neq 0$, to safely isolate the overflow infected population. The only feasible solution is hospitalizing at the optimal rates $\left(\gamma_{1}^{*}, \gamma_{2}^{*}\right)=(0.77,0.33)$ with $\phi=0$. This option requires isolating asymptomatic infectives faster than symptomatic infectives, a strategy which requires perfect hygiene and sanitary measures. This requirement cannot be met in sub-Sahara Africa given the state of the economies.

To illustrate the impact of non adherence to disease prevention measures on the optimal case in Figure 13, we varied the parameter $\alpha_{3}$. Figure 14 shows how the optimal solution in Figure 13 is altered by varying $\alpha_{3}$. The hospital bed capacity is exceeded for any case $\alpha_{3} \neq 0$. For the case $\phi \neq 0$, no matter how small $\phi$ is, no optimal strategy exists for the three provinces of
South Africa. It is unrealistic to expect perfect adherence to social distancing, wearing of masks and lockdown measures, in a region where the population survives on less than $\$ 1$ per day and there are no food banks.

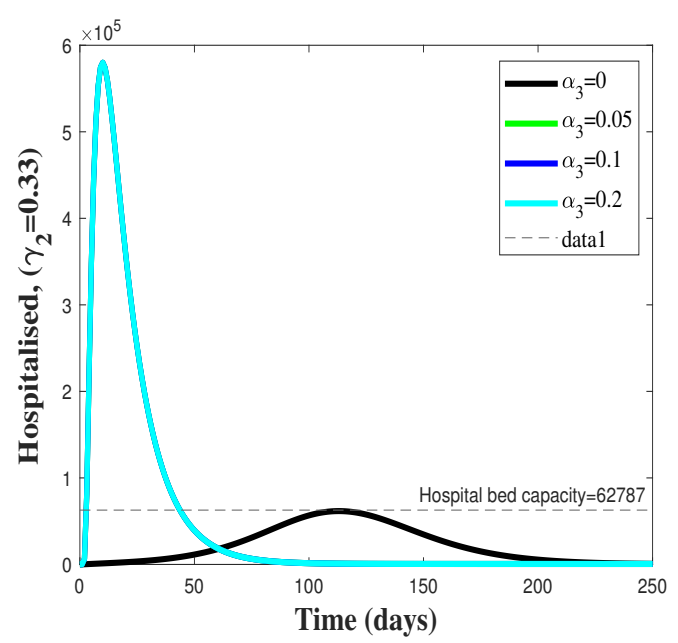

Fig. 14: Population level plots for hospitalized individuals for varying values of $\alpha_{3}$

2) Effect of early lockdown: South Africa introduced the lockdown very early. The strategy discussed in section $C .1$ is therefore not relevant. Hence, we consider the modified system with control given in $(19)-(25)$ and $(28)-(34)$. We consider hypothetically how the lockdown could have altered a non optimal strategy in Figure 13. We chose $\gamma_{1}=0.6$ and $\gamma_{2}=0.33\left(u_{1}^{*} \geq\right.$ $0.1, u_{2}^{*} \geq 0.1$ ) to illustrate this example (see Figures $15(a)-15(e))$.

The number of deaths for a model with controls for South Africa for different optimal cases of $\gamma_{1}$ and $\gamma_{2}$ is given in Table $V$. Table $V$ compares the actual number of recorded deaths, the controlled number of deaths and the uncontrolled number of deaths (no lockdown). It is clear that the lockdown was very effective.

\section{CONCLUSION}

The analysis has revealed that there are several strategies that can flatten the infection curve and protect hospital bed capacity. However, we have 
M H Machingauta, B Lungu, E M Lungu, COVID-19 changing the face of the world. Can sub-Sahara ...

TABLE IV: Number of people who must be hospitalized.

\begin{tabular}{|c|c|c|c|c|c|}
\hline$\gamma_{1}$ & $\gamma_{2}$ & $H$ & $H \times 1.5$ & $H$ peak $\left(H_{P}\right)$ & Oveflow \\
\hline 0 & 0.33 & 62787 & 94180 & 246174 & 151994 \\
\hline 0.3 & 0.33 & 62787 & 94180 & 207654 & 113474 \\
\hline 0.6 & 0.33 & 62787 & 94180 & 112952 & 18772 \\
\hline
\end{tabular}
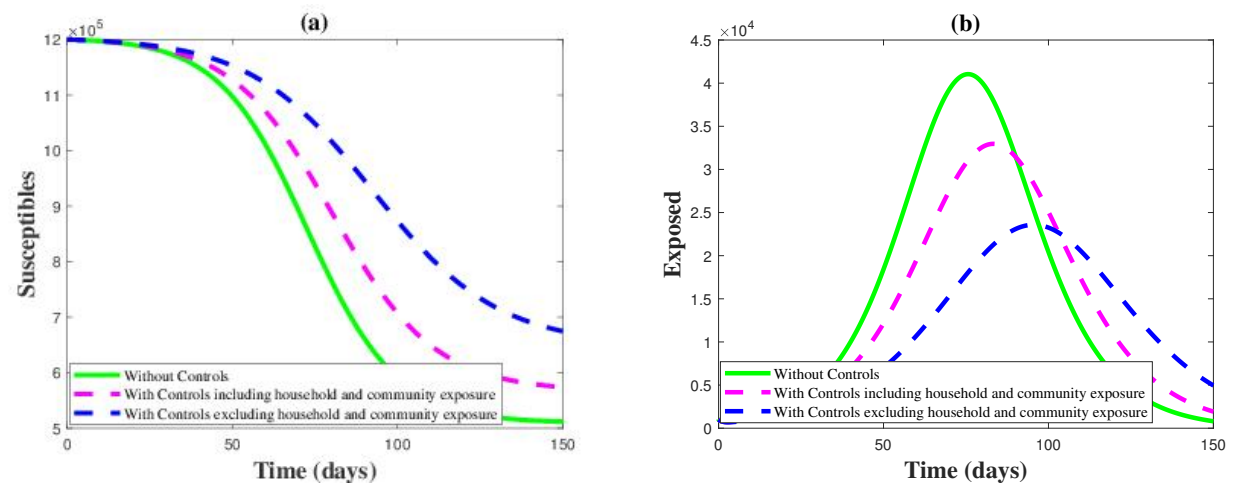

(c)
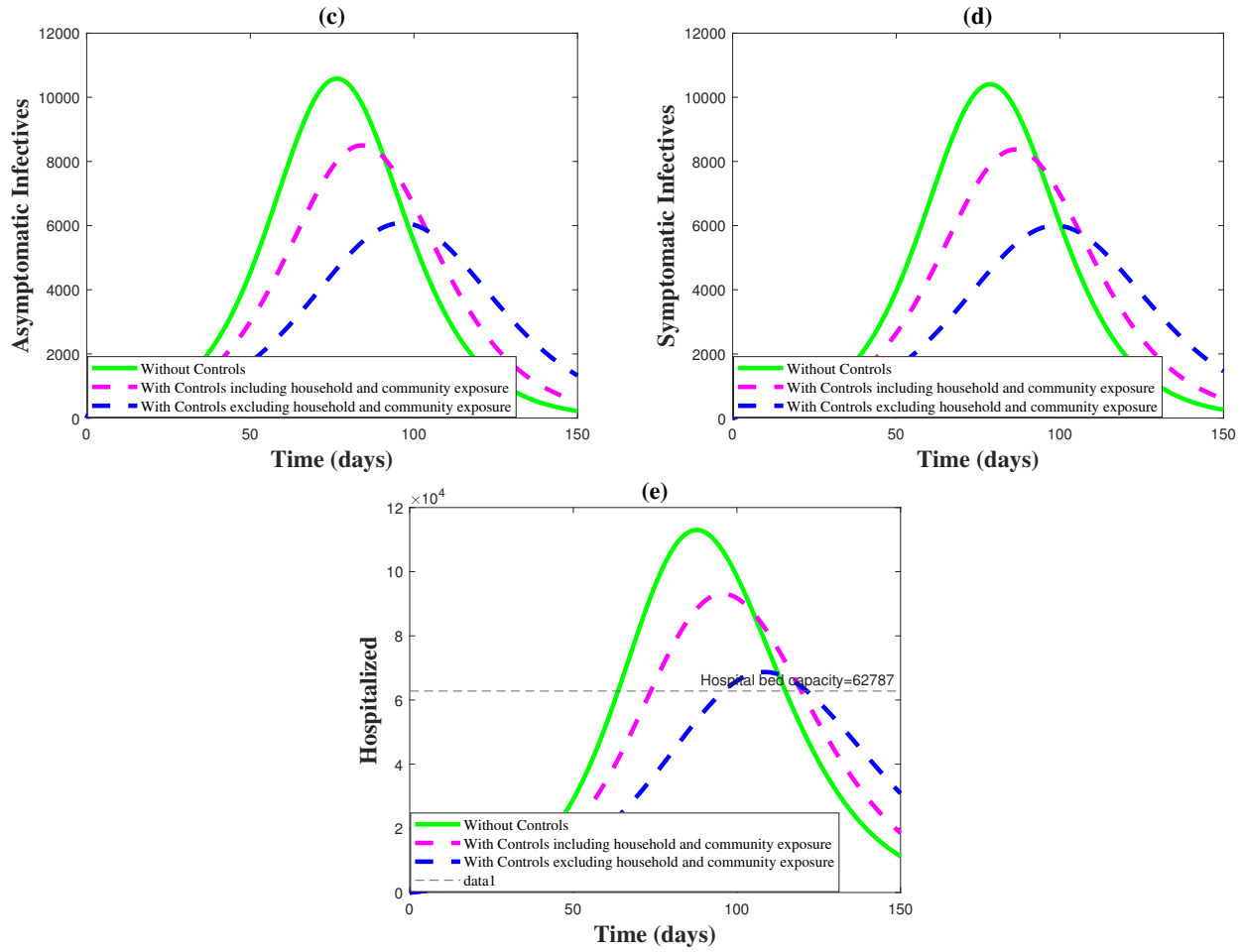

Fig. 15: A comparison of state variables with and without controls

found that of all the strategies, the preferred strategy is either that which removes asymptomatic infectives much faster than the symptomatic infectives or the strategy that removes symptomatic infectives faster than asymptomatic infectives as either of these strategies gives the least number of deaths.

The optimal control analysis suggests that if Italy had introduced the lockdown much earlier the number of deaths would have been reduced 
M H Machingauta, B Lungu, E M Lungu, COVID-19 changing the face of the world. Can sub-Sahara ...

TABLE V: Comparison of deaths at different optimal cases

\begin{tabular}{|c|c|c|c|c|c|c|}
\hline$\gamma_{1}$ & $\gamma_{2}$ & & 30 days & 60 days & 72 days & 90 days \\
& & & $\delta=0.003$ & $\delta=0.01$ & $\delta=0.01$ & $\delta=0.01$ \\
\hline 0.77 & \multirow{2}{*}{0.33} & no lockdown & 178 & 3358 & 5930 & 12394 \\
& & lockdown & 7 & 151 & 211 & 327 \\
& & actual & $(5)$ & $(123)$ & $(238)$ & $(705)$ \\
\hline 0.4 & \multirow{2}{*}{0.6} & no lockdown & 187 & 3805 & 6834 & 14287 \\
& & lockdown & 7 & 153 & 216 & 339 \\
& & actual & $(5)$ & $(123)$ & $(238)$ & $(705)$ \\
\hline
\end{tabular}

significantly and the hospital bed capacity would have been protected.

South Africa introduced the lockdown early. This is evident from the flatness of the infection curve (Figure 12). It is obvious from Fig.12 that early lockdown slowed down the number of infections. The question is why despite this measure the number of infected individuals has kept on rising. The number of infections in fact rose rapidly after the lockdown. It is clear from Figure 15 that the lockdown was ineffective because the individuals in various households came out to mingle with individuals from other households at community places such as shops, markets etc. The lockdown did not have the desired effect. From Figure 15 it is evident that if household and community transmission had been avoided due to higher levels of adherence to control measures the situation would have resulted in $33 \%$ fewer asymptomatic infectives, $33 \%$ fewer symptomatic infectives and $62 \%$ less hospitalizations.

The difficulty for South Africa, and indeed any sub-Sahara African country, will be how to ensure the populations living in high density areas observe social distancing and good hygiene practices. It is easier in developed countries with social security arrangements to maintain disease prevention measures. In sub-Sahara Africa where the majority of the people live a subsistence life and are self employed, it will be hard to enforce the measures employed in developed countries.

\section{Declarations of competing interest: None.}

This research did not receive any specific grant from funding agencies in the public, commercial, or not-for-profit sectors.

We thank the Simons Foundation (US) through
The Research and Graduate Studies in Mathematics (RGSMA) project at the Botswana International University of Science and Technology (BIUST) and the Department of Mathematics and Statistical Sciences of BIUST for their support.

\section{REFERENCES}

[1] Africa News (2020). South Africa coronavirus: US gives 1,000 ventilators; cases pass 11,000. Retrieved from https://www.africanews.com/2020/05/12/south-africacoronavirus-daily-updates-on-covid-19/ on 13 May 2020.

[2] Berge, T., Lubuma, M. S., Moremedi, G. M., Morris, N., \& Kaondera-Shava, R. (2017). A simple mathematical model for Ebola in Africa. Journal of Biological Dynamics, 11 : 1,42 - 74, DOI:10.1080/1753758.2016.12229817

[3] Collins, G. L., Haines, C., Perkel, R., \& Enck, R. E. (2007). Lung Cancer: Diagnosis and Management. American Family Physician, Jan 2007 Volume 75 Number 1

[4] Dell, A. J., \& Kahn D. (2017). Geographical maldistibution of surgical resources in South Africa: a review of the number of hospitals, hospital beds and surgical beds. S.Afri Med J 2017;107(12) : 1099 1105.DOI:10.7196/SAMJ2017.v107i12.12539

[5] Deressa, C.T., \& Duressa, G.F.(2020). Modeling and optimal control of transmission dynamics of COVID19: The case of Ethiopia. Alexandria Eng. J (2020), https:doi.org/10.1016/j.aej.2020.10.004

[6] FRANCE 24. (2020). French lockdown comes into force in bid to curtail spread of deadly virus. Retrieved from https://www.france24.com/en/20200317-frenchlockdown-comes-into-force-in-bid-to-curtail-spread-ofdeadly-virus on 25 April 2020.

[7] Friedman, A., \& Lungu, E. M. (2013). Can Malaria parasite pathogenesis be prevented by treatment with tumor necrosis factor-alpha. Mathematical Biosciences, 2013, 10(3): 609 - 624, doi:10.3934/mbe.2013.10.609

[8] Garba, S.M., Lubuma, J.M.S, \& Tsanou, B. (2020). Modeling the transmission dynamics of the COVID-19 Pandemic in South africa.Mathematical Biosciences 328(2020)108441, https://doi.org:10.1016/j.mbs2020.108441 
[9] Guan, W. J., Ni, Z. Y., Hu, Y. et al. (2020). Clinical characteristics of coronavirus disease 2019 in China. The New England Journal of Medicine. 2020

[10] Kamgang, J.C., \& Sallet, G. (2008). Computation of threshold conditions for epidemiological models and global stability of the disease-free equilibrium (DFE). Math Biosci 2008; 213 : 1 - 12

[11] Kassa, S.M., Njagarah, J.B.H, \& Terefe, Y.A. (2020). Analysis of the mitigation strategies for COVID-19: From mathematical modelling perspective.Chaos, Solitons and Fractals 138(2020)109968.

[12] Kolaye, G., Bowong, T. S., Houe, N. R, Aziz-Alaoui, M. A., \& Cadivel, M. (2019). Mathematical assessment of the role of environmental factors on the dynamical transmission of cholera. Communications in Nonlinear Science and Numerical Simulation;67 : $203-$ 222.,ISSN1007 - 5704 .

[13] Kombe, I. K., Munywoki, P. K., Baguelin, M., Nokes, J. D., \& Medley, G. F. (2019). Modelbased estimates of transmission of respiratory synctial virus within households. Epidemics 27 (2019) 1 - 11. https://doi.org/10.1016/j.epidem.2018.12.001

[14] Lenhart, S., \& Workman, J.T. Optimal Control Applied to Biological Problems, Chapman \& Hall/CRC, Boca Raton, Fla, USA, 2007.

[15] Levy, B., Edholm, C., Gaoue, O., Kaondera-Shava, R., Kgosimore, M., Lenhart, S., Lephodisa, B., Lungu, E., Marijani, T., \& Nyabadza, F. (2017). Modeling the role of public health education in Ebola virus disease outbreaks in Sudan.Infectious Disease Modelling 2(2017) 323 340.

[16] McKinsey \& Company. (2020). Covid19:Briefing materials. Global health and crisis response, March 25, 2020. Retrieved from https://www.mckinsey.com//media/McKinsey/

BusinessFunctions/Risk/OurInsights/Covid19Implications forbusiness/Covid19March30/COVID-19-Facts-andInsights-March-25-v5.ashx on 22 April 2020.

[17] Medecins Sans Frontier (MSF). (2020). New Ebola cases confirmed in DRC days before expected end of outbreak. Project update 23 April 2020. Retrieved from https://www.msf.org/new-ebola-cases-confirmed-drc on 9 May 2020.

[18] Ngeleja, R.C., Luboobi, L. \& Nkansah-Gyekye, Y. (2016) Stability Analysis of Bubonic Plague Model with the Causing Pathogen Yersinia pestis in the Environment. Advances in Infectious Diseases, 6, 120 137.http://dx.doi.org/10.4236/aid.2016.6301.

[19] Ngoghala, C.N., Iboi, E., Eikenberg, S., Scotch, M., McIntyre, C. R., Bonds, M. H., \& Gumel, A. B. (2020). Mathematical assessment of the impact of non-pharmaceutical interventions on curtailing the 2019 novel coronavirus. Mathematical Biosciences:325(2020)108364.

[20] Mukandawire, Z., Nyabadza, F., Malunguza, N. J., Cuadros, D. F., Shiri, T., \& Musuka, G. (2020). Quantifying early COVID-19 outbreak transmission in South Africa and exploring vaccine efficacy scenarios. PloS ONE:15(7) : e0236003, https://doi.org/10.1371/journal.pone0236003.

[21] Peters, J. (2020). All of Italy is being placed on lockdown due to coronavirus. It's an expansion of LOmbardy's lockdown announced yesterday. Retrieved from https://www.the vererge.com/2020/3/9/21172390/italylockdown-coronavirus-expansion-lombardy on 25 April 2020.

[22] Pulla, P. (2020). What counts as a covid-19 death? BMJ 2020;370:m2859. doi:https://doi.org.10.1136/bmj.m2859.

[23] Rhodes, A., Ferdinande, P., Flaatten, H., Guidet, P., Meitnitz, P. G., \& Moreno R. P. (2012). The variability of critical care bed numbers in Europe.Intensive Care Med (2012):38(10):1647-53. DOI:10.10007/s00134-012$2627-8$.

[24] Rodriguez-Llanes, J. M., Castro Delgado, R., Pederson, M. g., Arcos Gonzáles, P., \& Meneghini, M. (2020). Surging critical care capacity in Italy. [Submitted]. Bull World Health Organ. E-pub:6 April 2020.http://dx.doi.org/10.2471/BLT.20.257766

[25] Rothan, H. A., \& Byrareddy, S. N. (2020). The epidemiology and pathogenesis of coronavirus disease (COVID-19) outbreak. Journal of Autoimmunity. https://doi.org/10.1016/j.jaut.2020.10.2433.

[26] Salwani, S. (2017). Covid-19: Africa told to prepare for the worst. what's the response? Retrieved from https://www.aljazeera.com/news/2020/03/covid19-africa-told-prepare-worst-response200319085112877.html on 25 April 2020.

[27] Sánchez Nicolás, E. (2020). Coronavirus: Spain’s lockdown will 'last more than 15 days'. Retrieved from https://euobserver.com/coronavirus/147763 on 25 April 2020

[28] Tang, B., Wang, X, Li, Q., Luigi Poragazzi, N., Tang, S., Xiao, Y \& Wu, J. (2020). Estimation of the transmission risk of the 2019-nCov and its implication for public health interventions. J.Clin.Med.2020, 9, 462;doi:10.3390/jcm9020462

[29] Van den Driessche, P. \& Watmough, J. (2002). Reproduction numbers and sub-threshold endemic equilibria for compartmental models of disease transmission. Mathematical Biosciences, vol. 180, $29-48$.

[30] Veeranna, C. H., \& Rani, S. (2020). Cause of death certification in COVID-19 deaths. Indian J Crit Care Med 2020 Sep; 24(9) : 863 - 867, doi:10.5005/jp-journals$10071-23561$.

[31] Wang, D. R., Hu, B., Hu, C., Zhu, F., Liu, X., Zhang, J., Wang, B., Xiang, H., Cheng, Z., Xiong, Y., Zhao, Y., Li, Y., Wang, X., \& Peng, Z. (2020). Clinical characteristics of 138 hospitalised patients with 2019 novel coronavirus-infected pneumonia in Wuhan, China. JAMA 2020,doi:10.1001/jama.2020.1585.

[32] World Health Organisation. (2011). Health situation analysis in the African region. atlas of health statistics, 2011. Retrieved from 
M H Machingauta, B Lungu, E M Lungu, COVID-19 changing the face of the world. Can sub-Sahara ...

https://apps.www.who.int/medicinedocs/documents/ s18863en/s11863en.pdf on 7 April 2020.

[33] World Health Organisation. (2020). Rolling updates on coronavirus disease (COVID-19). Retrieved from https://www.who.int/emergencies/diseases/novelcoronavirus-2019/events-as-they-happen on 23 March 2020.

[34] World

Odometer.

(2020).

COVID-19 coronaviruss pandemic. Retrieved from https://www.worldodometersinfo/coronavirus/2utmcampaign $=$ homeAdUOa?Si on 20 January 2021.

[35] Worldbank. (2020). The World Bank life expectancy at birth, total (years) - Italy. Retrieved from https://data.worldbank.org/indicator/SP.DYN.LE00IN? locations=IT on 29 June 2020.

[36] Worldbank. (2020). The World Bank life expectancy at birth, total (years) - South Africa. Retrieved from https://data.worldbank.org/indicator/SP.DYN.LE00IN? locations=ZA on 29 June 2020.

[37] Wu, C., Chen, X., Cai, Y. et al. (2020). Risk factors associated with acute respiratory distress syndrome and death in patients with coronavirus disease 2019. Pneumonia in Wuhan, China JAMA Intern Med. 2020 